\title{
Supporting Decision Making in Engineering Design using Parallel Coordinates in Virtual Reality
}

\author{
Sławomir K. Tadeja* and Timoleon Kipouros ${ }^{\dagger}$ and Yupu $\mathrm{Lu}^{\ddagger}$ and Per Ola Kristensson ${ }^{\S}$ \\ Department of Engineering, University of Cambridge, Cambridge, U.K. \\ Faculty of Physics, Astronomy and Applied Computer Science, Jagiellonian University
}

\begin{abstract}
Computational engineering design methods and tools are common practices in the modern industry. Such approaches are integral in enabling designers to efficiently explore large and complex design spaces. However, they also tend to dramatically increase the number of candidate solutions that decision makers must correctly interpret. Since all candidate solutions can be represented in a digital form together with their assessment criteria, a natural way to explore and understand the complexities of the design problem is to visualize their multidimensional nature. The task now involves the discovery of patterns and trends within a multidimensional design space. In this work, we aim to enhance the design decision making process with immersive Parallel Coordinates Plot (IPCP) in virtual reality. We present the design of this system, which allows representation and exploration of multidimensional scientific datasets. A qualitative validation with two surrogate expert users demonstrated that the system can be used successfully to both detect both known and previously unknown patterns and support learning the decision making process in a shorter time. The results serve as a promising indication of how immersive parallel coordinate plots can enhance decision support in complex engineering design processes.
\end{abstract}

\section{Introduction}

Computational design methodologies and customized optimization tools are common practice in modern engineering. The primary benefit of such approaches is the automated exploration of complex design spaces, which was previously not possible. However, this advantage creates user interface challenges on how to support manipulation and analysis of high volume and high complexity multidimensional datasets. While even formulating a real-world engineering optimization problem is a difficult task, the inherent additional difficulties in extracting understanding, causality, and rationale of discovered information is exacerbating the demands on the user.

The ultimate goal in this work is to assist users in understanding and then communicating the complexities of the engineering design task in question and thereby supporting the decision making process. As a step towards this goal,

\footnotetext{
*PhD Student, Engineering Design Centre, Department of Engineering, University of Cambridge, skt40@eng.cam.ac.uk, and Assistant, Faculty of Physics, Astronomy and Applied Computer Science, Jagiellonian University, slawomir.tadeja@uj . edu.pl

†Senior Research Associate, Engineering Design Centre, Department of Engineering, University of Cambridge, t. kipouros@eng.cam. ac .uk

$¥$ Visiting Student, Engineering Design Centre, Department of Engineering, University of Cambridge, yl737@cam. ac.uk

${ }^{\S}$ Professor, Engineering Design Centre, Department of Engineering, University of Cambridge, pok21@eng.cam. ac.uk.
} 
this paper presents the system design of an immersive version of the well-established Parallel Coordinates Plots (IPCP). Prior work [1] has demonstrated that visualizing scientific information in an interactive and engaging environment is beneficial to users' decision making abilities. We hypothesize that further benefits of visualization can be realized using emerging 3D immersive environments, such as the one offered by Virtual Reality (VR).

In this work, we explore how VR can be used to support the visualization and analysis of computational engineering design datasets. In addition, we discuss the positive and negative aspects of the application of VR in the decision making process. The overall task we study is the identification of clusters and sets of solutions in two optimization studies for the aerodynamic design of compressor blades [2, 3], and the design of S-ducts [4]. However, we do not consider the physical interpretation and understanding of the designed compressor blades. We rather consider the physical nature of multi-objective optimization data. In such datasets, the input parameters express the design variables of the design optimization study, and the output parameters express the objective functions and constraints.

In this paper, we do not address the classic challenges in using PCP for visualization and analysis of multidimensional scientific data. Instead, we explore how to build a system that can support the design process and investigate the usability of emerging immersive technologies for visualization of, and interaction with, scientific information. This allows us to assess which challenges remain, or new ones emerge. Furthermore, the exploration of engineering design computational data is frequently centered on 3D visualization of the designed system. As such, an immersive interface offers a highly promising method for analysis and exploration of such data alongside with simultaneous visualizations of individual product designs. Part of the motivation of this work is to expose and exploit such strengths of VR technology such us the possibility of immersing the user into bespoke, 3D environment fully simulated by the system's designer.

To this end, we present the design of our VR-based IPCP system that is supported by a series of formative studies. A description of the first thereof which briefly discusses the observational user study, the results of which we used to trim the vast design space, can be found in Tadeja et al. [5, 6]. The underpinning VR system that included the basis of interaction techniques i.e. the gaze-tracking and ray-tracing facilitated with a cross-hair metaphor was discussed in Tadeja et al. [7, 8] and is built based on the Unity VR Samples Pack [9] that utilizes the well-known concept of a cross-hair to simulate the user's gaze (for instance, see the orange circle in Fig. 5] and Oculus Utilities for Unity [10] together with some commercial assets from the Unity asset store packages [11].

\section{Immersive Parallel Coordinates}

Since the PCP concept was initially proposed by Inselberg [12] the number of entries containing the phrase "parallel coordinates", as returned by Google Scholar search on December $9^{\text {th }} 2020$, reaches over 2.030 million items. The theory and applications of PCP can be found in the textbook written by Inselberg [13].

The wide adoption of 2D PCP has led to multiple attempts of designing a useful and compelling 3D PCP. For example, Wegenkittl et al. [14] and Gröller et al. [15] describe Extruded PCP, which uses a PCP to visualize a complex 
surface. A different example of using the surface-type design is a density isosurface [16], which was used to visualize cytomics data. Falkman [17] describes the Cube package applied for swift analysis of medical data.

Parallel Glyphs combine Star Glyphs [18] with 3D PCP [19]. The usability of 3D Multi-Relational PCP in conjunction with High-Precision Textures has been investigated in Johansson et al. [20]. Dang et al. [21] describe a few examples of 3D parallel dot displays and stacked PCP, which uses various stacking techniques to augment the standard visualization of dot plots and PCP respectively. In their related work, Chang et al. [22] compared joined side-by-side 2D visualizations to PCP with scatter plot matrices, concluding that the PCP-based approach offered clear advantages over individual visualizations for most of their participants for a number of their tasks. Furthermore, Johansson et al. [23] presented a comparison between 3D PCP visualizations with conventional 2D plots and concluded in favor of the latter. Holten et al. [24] discuss different variants and improvements of 2D PCP used to find clusters in the multivariate datasets and remarks that only the use of additional scatter plots has a substantial positive impact on the visualization.

ImAxes [25] allows the user to rearrange the axes interactively and supports both 2D and 3D scatter plots. Another example of an immersive PCP is the ART collaborative data analysis tool [26] that distributes the user interface across an augmented reality headset and a touch surface. Rosenbaum et al. [27] took another approach in which the user was treated as an element of the immersive PCP visualization itself.

Our approach, first described in Tadeja et al. [5] and later on discussed in Tadeja et al. [6], differs from other attempts in several key ways. First, we denote values in each dimension with interactive volumetric objects (i.e., unit-size cubes) that automatically highlight the item under the user's gaze in order to signal that they can be interactive. The same is true for all the other interactive elements, such as selectors (i.e., various size spherical markers in the interface) used to move or rotate the scatter plots. This in turn opens up new design possibilities [28] that can use characteristics of a 3D shape, such as its orientation in the 3D space [28]. In addition, users can freely move within and maneuver in the "infinite" 3D space to explore all parts of the visualization [5]. Furthermore, our system augments the pattern identification task with 3D scatter plots [5- $[$ ], that can substantially aid users in isolating groups of data items that can potentially form patterns. In addition, in this paper, we are also exploring a new way of the bimanual user interaction method. Here, we are investigating the feasibility of using a gesture-based interface facilitated with an additional sensor [29] attached to the VR headset that tracks the user's hands in real-time.

\section{Problem Context}

We emphasize that the purpose of the two case studies is not only to demonstrate how to identify patterns in high dimensional engineering datasets, but also to demonstrate the effectiveness and usefulness of the utilization of VR technology in the discovery of meaningful information in complex datasets with ease and with common intuition. Both cases represent real-world design problems in the field of turbomachinery.

In a typical process, when an automated shape aerodynamic design optimization process is executed successfully, the 

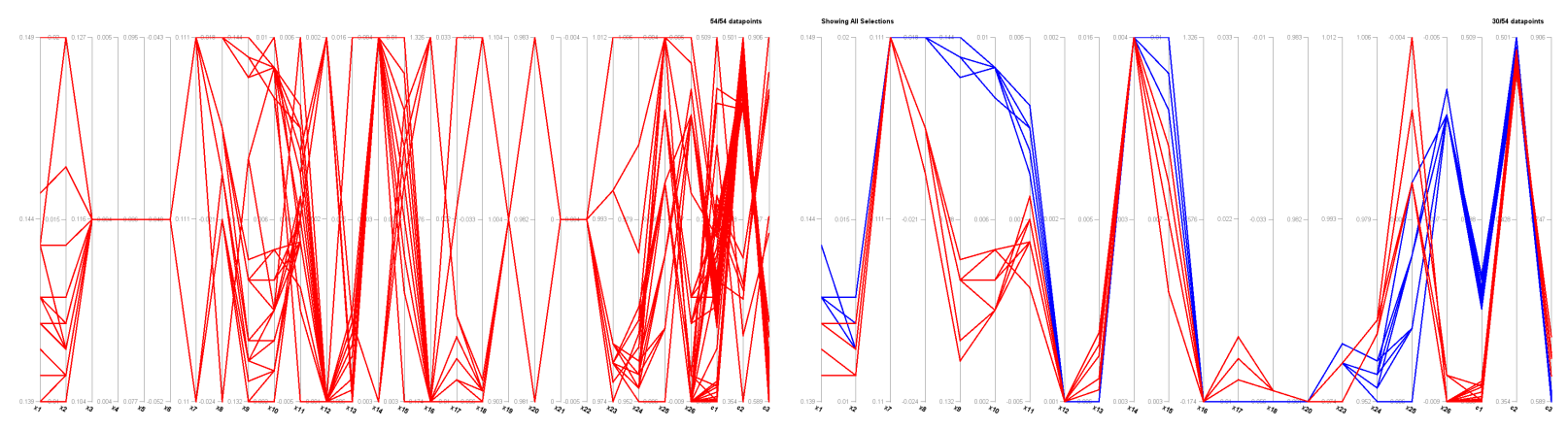

Fig. 1 The left plot shows the complete dataset in the standard 2D PCP. The two patterns are shown in red and blue on the right plot. Reproduced from Kipouros et al. [1].

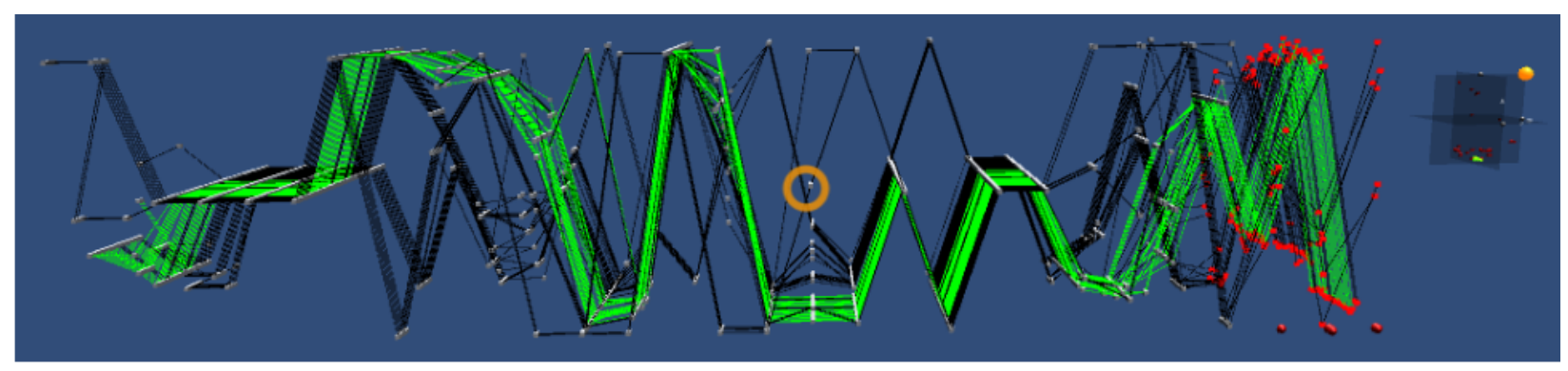

Fig. 2 The user's field of view of the 29-dimensional dataset of 54 equally optimum design configurations describing the Pareto front (26 design parameters and 3 objective functions).

designer discovers a set of solutions representing the answer to the multi-criteria design problem. This set of solutions, the Pareto set, is an approximation of the Pareto front. In order to achieve this, the automated optimization process should synthesize three modules. One to modify the geometry, and is associated with the design variables, the second to simulate the flow characteristics, and is associated with the quantification of the objective functions, and the third to navigate through and explore the design space, which is the optimization algorithm. Prior work [1] introduced a stage of post-processing of optimization results where the user simultaneously considers the design parameters and objective functions. Depending on the complexity of the optimization problem, the number of solutions within the Pareto set can easily exceed a few hundreds. The objective at this stage of the design process is to identify a few families, or groups, of solutions to aid the decision making process. The different groups of solutions are identified as clusters within the multidimensional data space. It is this final stage that we enhance in this work with the utilization of VR-based IPCP.

The first sample dataset is the result of an optimization study for the 3D geometrical design of compressor blades subject to many equality and inequality physical and geometrical constraints. In this study, we use the data produced for a three-objective function formulation of the optimization problem as described in detail in Kipouros et al. [2, 3]. This dataset (hereafter referred to as DS1) expresses the Pareto front, which contains a set of 54 equally optimum design configurations, and the 29-dimensional data items categorized as: design parameters (26 dimensions) and objective functions ( 3 dimensions). The 2D view of the complete dataset, as well as the two patterns which were identified using 
the conventional 2D PCP, are presented in Fig. 1. The two patterns of interest $A$ (in red) and $B$ (in blue) are composed of 11 and 19 data items respectively. These two patterns were identified by domain experts and we consider them as the reference in this work. The user's view of the same dataset through a VR headset in 3D is shown in Fig. 2

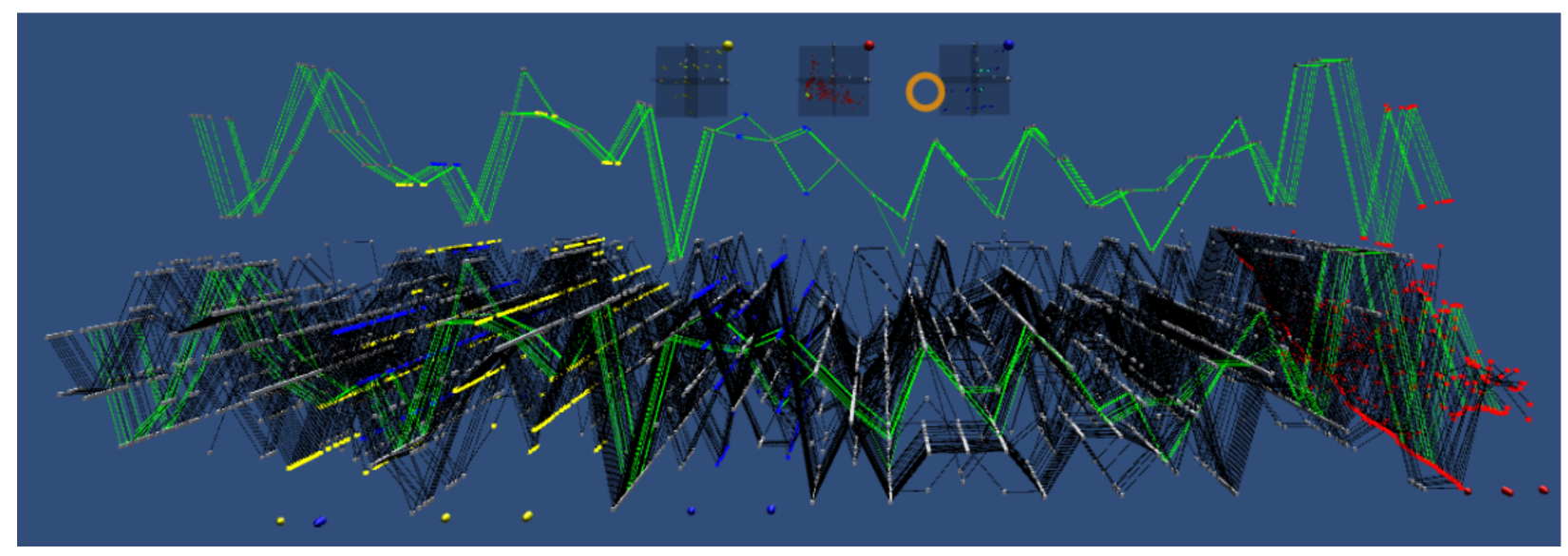

Fig. 3 The user's field of view of the 39-dimensional dataset (36 design parameters from FFD parameterization and 3 design criteria: CP, DC60, and Swirl).

The second sample dataset (hereafter referred to as DS2) describes the results of the optimization study of S-Duct design (see Fig. 8), as presented in D'Ambros et al. [4] and is composed of 39-dimensional data items: design parameters (36 dimensions) and objective functions (3 dimensions). The user's view through a VR headset in 3D is presented in Fig. 3 .

\section{System Design}

\section{A. Overview}

The overall objective is to design a visual analytics IPCP system for engineering design processes, supporting efficient identification and comparisons of patterns of interest in a multidimensional dataset. This is a high-dimensional design space with many controllable and uncontrollable parameters. Controllable parameters include rendering parameters, such as colors and shapes, visual/auditory feedback design, interaction techniques, and choice of headset and hardware controllers. Uncontrollable parameters include properties about the datasets, such as size, density, and correlation structure, and the degree of domain knowledge and expertise of the user.

As a result of the many controllable and uncontrollable parameters, we have adopted an iterative process of first building an early system that provided direct transplantation of PCP to VR. This early design was then used to carry out qualitative user studies (partially described in a previous poster publication Tadeja et al. [5]), briefly outlined in subsection IV|IV.B. We use the information distilled from the user study along with knowledge of the tasks, expert advice, and technical constraints to arrive at a set of design principles (see IVIV.E), which guide our task analysis in IV IV.F In the task analysis, we break down each task into functions and translate each function into a solution 


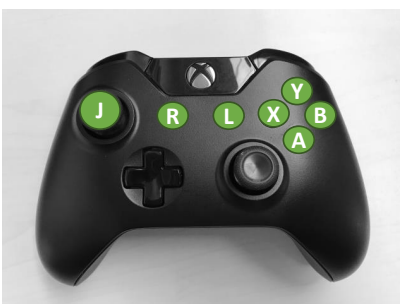

(a)

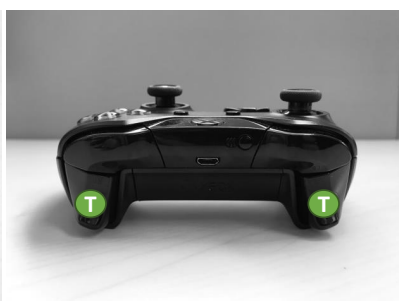

(b)

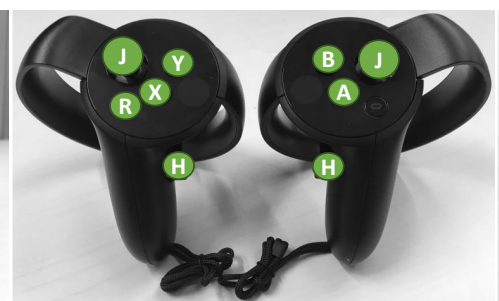

(c)

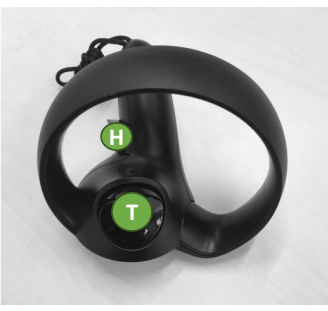

(d)

Fig. 4 VR controllers : (a-b) an Xbox gamepad; (c-d) Oculus Touch. The green markers are showing the mapping between the action-buttons on both types of controllers.

based on technical constraints, expert advice, and information gathered from the qualitative user study[5]. Finally, we provide verification and validation of the IPCP system in section $\mathrm{V}$ whereas section VII provides a description of the implemented extensions to the system. These are twofold. First, we couple the system with Computer-Aided-Design (CAD) 3D models of the actual geometries of the individual designs associated with individual data items on the IPCP. Second, we developed and implement a different manipulation technique that utilizes the hand-tracking and gesture-recognition done with the help of Leap Motion Controller [29] coupled with gaze-tracking to aid the user in more effective interaction with our system. Finally, in section VIII we provide a detailed discussion about the entire process of design, development, and validation of the IPCP system. Whereas, section $[\mathrm{IX}]$ offers a brief summary of our work.

\section{B. Qualitative Study}

To help inform the design we carried out a qualitative study with seven participants (hereafter referred to as P1-P7). More details about this study can be found in Tadeja et al. [5]. The IPCP visualization used in the study can be seen in Fig. 2 and Fig. 3 . The users could interact with the system using a mixture of gaze-tracking and Xbox gamepad

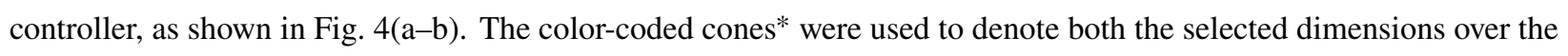
IPCP and axes directions at the scatter plots [7, 8], see Fig. 4(a, c).

Although the visualized dataset comes directly from the specific domain — the aerodynamic design of turbomachinery components-we wanted to pinpoint the general underlying design principles that can be used in future more advanced versions of the IPCP. Therefore, we broadened our sample of participants to include both fields of expertise and prior familiarity with PCP. The volunteers self-assessed their level of expertise with regards to VR and PCP using a Likert-like scale. As the color-coding plays a crucial role in the visualization, participants were pre-screened with Ishihara's tests [30], which indicated no color deficiency.

On average, participants needed 25 minutes during the training phase and 15 minutes for the study itself. To offer insight into any adverse physiological effects, volunteers were instructed to take the simulation sickness questionnaire

\footnotetext{
*W. Kresse, used under CC BY-SA 3.0; http://wiki. unity3d.com/
} 
(SSQ) [31] before and after each phase (i.e., training or study) to see to what extent and if at all the visualization impacted negatively on their perception of comfort. Participants were reminded that they should stop the experiment if they experienced any of the symptoms described in the survey. However, none of the participants decided to stop and discontinue the study.

Before the experiment commenced, each participant sat through a demonstration of the IPCP technique, the studied tool, and all the possible ways in which the user could interact with the system. Moreover, while listening to a verbal description, participants could track in real-time the instructor's actions and their corresponding results on a computer screen.

Our participants reported having varying levels of prior familiarity with the PCP and VR. To level the playing field, we provided each volunteer with training data visualization. This process took between 18 and 36 min, depending on the individual participant's needs as judged by the researcher. The primary task of the user was to find patterns within the given dataset using IPCP. In this context, the pattern is understood as a group of data items sharing similar values across all the dimensions visualized as group of polygonal curves (see Fig. 1 1 left)). However, such curves can have certain variability between one or more of the line segments. The level of this variance is left to be judged by the user.

This primary task was split into subtasks, including: a) selection of both data items and dimensions on the PCP; b) selection of data points and clusters on the scatter plots; c) visual analysis of the relation between the visualization elements; and d) rotation and movement of a scatter plot or generation of more than a single such 3D projection at once. Familiarization with the system was especially important, since the training may be very beneficial for the participant's grasp of the PCP technique, as remarked by Shneiderman et al. [32]. For this purpose, we used a portion of a real-world dataset of 80 multidimensional data points, each consisting of 22 dimensions (20 inputs and 2 outputs). The training was followed up with a semi-structured interview. If deemed necessary by the researcher, the participant was asked to repeat previously completed tasks. Each instruction was marked as "pass" by the study supervisor who constantly monitored the participant's actions on the accompanying screen. During this process, the researcher provided oral instructions upon completion of a task, and whenever the participants requested assistance.

To minimize fatigue, participants were given a break of approximately $15 \mathrm{~min}$ after training and before the main experiment commenced. After training, the participants were asked to perform a task within the VR environment without a time constraint. The main task had an identical form compared to the training: identify patterns in a multidimensional dataset of 26 input parameters and 3 output criteria, together with the suggested approach to start developing the scatter plot using the criteria dimensions. We adopted a think-aloud protocol and requested participants to discuss what, why, and how they are trying to achieve as the task progressed. The supervisor, who continuously monitored the experiment, could at any moment inquire for further clarifications to gain more insight into the participant's actions and thought processes. In particular, for the first-time gamepad users, an inquiry on whether the participant is stuck allowed the supervisor to provide guidance, for example, information about which button to click to invoke a specific action. This is 


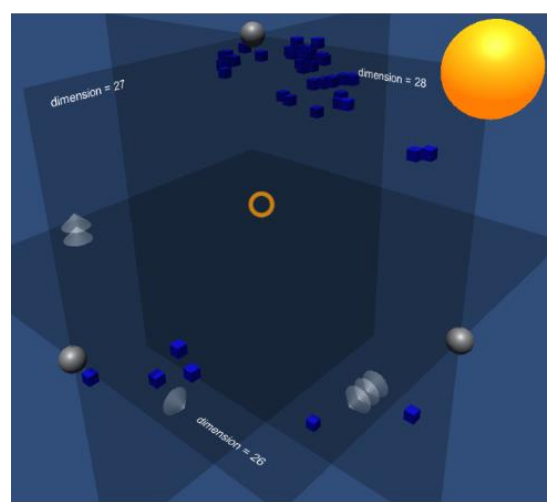

(a)

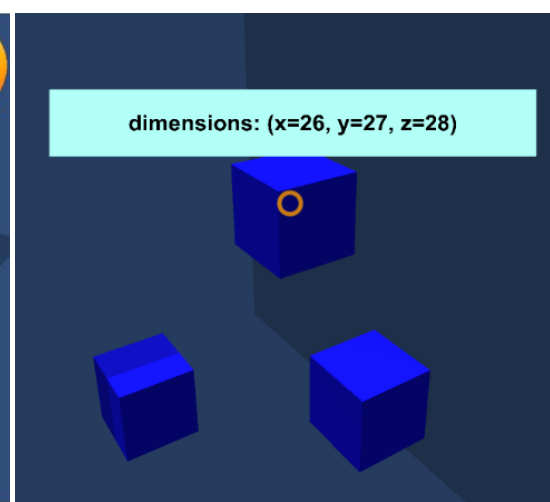

(b)

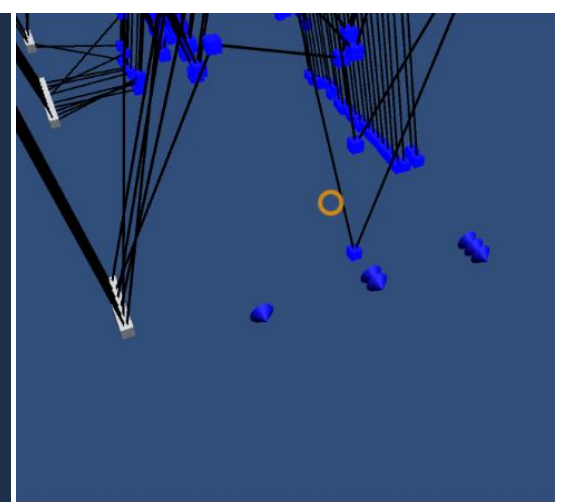

(c)

Fig. 5 (a) The 3D scatter plot [7, 8] generated from selected IPCP dimensions. (b) Zoomed-in volumetric data points. (c) The cones marking mapped dimensions on the IPCP.

important as the controller is not visible through the VR headset. Moreover, after running two participants (P1-P2), we decided to instruct the remaining participants (P3-P11) that a pattern is constituted by a minimum of a four-points group. Audio and video were captured during the study for later analysis.

\section{Participants' Background and Expertise}

All the participants reported having a background and extensive experience in the STEM (science, technology, engineering, and mathematics) fields in either industrial or academic settings or both. Nine participants were men and two were women with their age ranging between $22-44$.

Participant 1 (P1) reported approximately six years of experience in engineering at undergraduate and graduate levels and two years spent in the automotive industry. This person was not previously exposed to the PCP and had only very limited experience with VR.

Participant 2 (P2) reported less than three months of industrial experience. However, this volunteer has a background in aerospace engineering and engineering design spanning over nine years in total. This person self-rated as a novice in using VR and with even less familiarity with PCP.

Participant 3 (P3) spent seven years in an academic setting and almost a year in the industry. This person reported to have not previously used any VR headset. However, this participant is an expert on the PCP technique and its applications in design.

Participant 4 (P4) reported sixteen years of academic experience and three years of prior work in the industry. This participant is also an expert on PCP methods applied in modeling of the design processes.

Participant 5 (P5) has twenty-one years of academic (15 years) and industrial (6 years) experience in Computer Science and software engineering. This person also reported basic awareness with respect to VR and extensive exposure to PCP. 
Participant 6 (P6) has a background in Artificial Intelligence and Computer Science (9 years). During that time, this volunteer has also worked for three years with the PCP and multidimensional data visualization methods.

Participant 7 (P7) reported that he has an academic background in mathematics and computational fluid dynamics (8 years). However, this participant was a novice in terms of prior familiarity with VR and PCP.

Participant 8 (P8) was a doctoral student working on the application of analytical methods such us subspace-based dimension reduction to manufacturing deviations in turbomachinery components. This participant also self-reported having some limited experience with the VR and 2D PCP.

Participant 9 (P9) self-reported an intermediate level of familiarity concerning both 2D PCP and VR. P9 was also an expert in the field of mechanical and manufacturing engineering with vast experience of multi-objective optimization in academia and industry.

Participant 10 (P10) reported having some limited prior exposure to VR and advanced knowledge about the 2D PCP. This person used the 2D PCP for conducting research for the master's thesis in aerospace engineering. P10 also holds an undergraduate degree in the same area. In addition, this participant also recognized the dataset in which he looked for the patterns, as P10 used the same data for prior research involving 2D PCP. However, this person reported not spending a substantial amount of time on the analysis of this data, and P10 did not previously try to specifically identify the contained patterns.

Participant 11 (P11) self-reported to never before using the VR headset and to have some limited prior exposure to 2D PCP. This participant also reported to have undergraduate and postgraduate degrees in mechanical and aeronautical engineering and is currently in his first year of doctoral studies in aerospace engineering.

Table 1 The groups of data items identified as potential patterns by the participants (P1-P7). The color-coding of this data indicates the overlap with the two patterns (two bottom rows) identified by Kipouros et al. [1] as can be seen in Fig. 1 in red and blue respectively.

\begin{tabular}{|c|c|c|c|c|c|c|c|}
\hline $\begin{array}{c}\text { Pattern } \\
\text { candidate }\end{array}$ & P1 & P2 & P3 & $\mathrm{P} 4$ & P5 & P6 & P7 \\
\hline 1 & $\begin{array}{c}34,35,36 \\
38,39\end{array}$ & 2,5 & $\begin{array}{c}8,9,10,11, \\
12,13,14,15, \\
16,17,18,21, \\
22,23,24,25, \\
26,30\end{array}$ & $\begin{array}{c}8,9,10,11, \\
12,13,14,15, \\
16,17,18,21, \\
22,23,24,25, \\
26\end{array}$ & $34,37,38$ & $\begin{array}{c}33,34,35,36 \\
38,40,41 \\
42,43\end{array}$ & $\begin{array}{c}8,9,10,11, \\
12,13,14,15, \\
16,17,18,23, \\
24,25,26\end{array}$ \\
\hline 2 & $\begin{array}{c}8,9,10,12, \\
13,14,15,16, \\
17,18,23,24, \\
25,26,27,28\end{array}$ & 0,1 & $\begin{array}{c}33,34,35,36, \\
38,40,41 \\
42,43\end{array}$ & $\begin{array}{c}33,34,35,36, \\
38,40,41,42\end{array}$ & $6,8,9$ & $\begin{array}{c}8,9,10,11, \\
12,13,14,15, \\
16,17,18,21, \\
22,23,24,25, \\
26,30\end{array}$ & $\begin{array}{l}33,34,35, \\
36,38,40\end{array}$ \\
\hline 3 & $\begin{array}{c}9,10,12,13, \\
14,15,16,17, \\
18,24,25,26, \\
27,28\end{array}$ & $\begin{array}{c}7,9,10,17 \\
19,27,29,42\end{array}$ & $\begin{array}{c}47,48,50, \\
51,52,53\end{array}$ & $\begin{array}{c}8,9,10,11, \\
12,13,14,19 \\
20,21,22\end{array}$ & $29,30,39$ & $\begin{array}{c}8,9,10,11, \\
12,13,14,15, \\
16,17,18,23, \\
24,25,26,27, \\
28,29,30\end{array}$ & - \\
\hline 4 & - & 47,51 & - & - & - & $\begin{array}{c}46,47,48,49 \\
51,52,53\end{array}$ & - \\
\hline Pattern A & \multicolumn{7}{|c|}{$32,33,34,35,36,38,39,40,41,42,43$} \\
\hline Pattern B & \multicolumn{7}{|c|}{$8,9,10,11,12,13,14,15,16,17,18,23,24,25,26,27,28,29,30$} \\
\hline
\end{tabular}




\section{Identifying Patterns}

Kipouros et al. [1] provided an analysis that discovered two patterns of importance, shown in Fig. 11.b). As the study involved a real-world dataset (DS1), participants were instructed to note that some variability was to be expected in the data. However, they had to decide independently on what level of variability would be acceptable. Only two participants (P2 and P5) failed to recognize most of the data points belonging to the two groups as shown in Tab.1. In three cases, the participants changed their initial pattern candidates by selecting or deselecting elements originally grouped, thus creating subsets or partial repetitions of the previous selection (P1, P4, and P6). The majority of the participants identified almost the complete pattern $A$ (with completion ratios for P3:9/11, P4:8/11, P6:9/11 and P7:6/11) or $B$ (with completion ratios for P1:16/19, P3:16/19, P4:15/19, P6:19/19 and P7:15/19). These differences most likely come from limited knowledge of the area from which the dataset was sourced and, as such, the allowed levels of noise between the respective values.

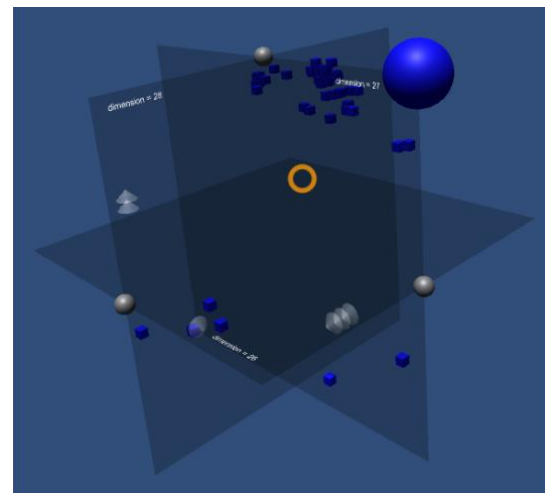

(a)

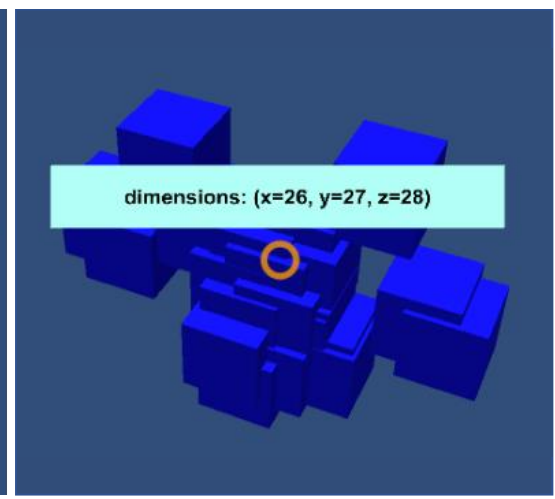

(b)

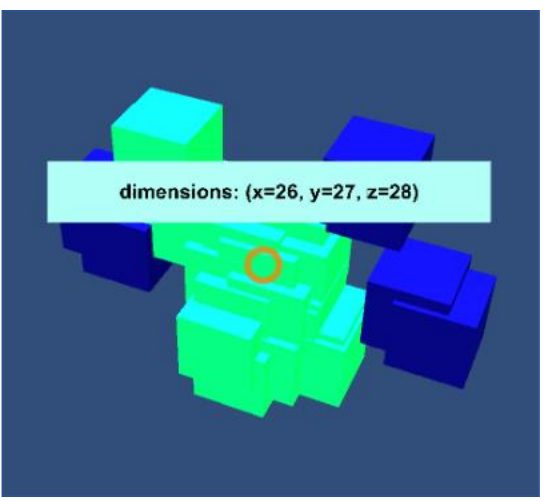

(c)

Fig. 6 (a) The 3D scatter plot $[7,8]$ and the results of a simple clustering algorithm applied to overlapping data points (b-c).

\section{Observed Behavior}

The results of the study involving the first seven participants (P1-P7) suggest that the time needed for the analysis was correlated with prior experience with PCP, that is, the more expertise the participant had, the longer it took to finish the training session. Simultaneously, experts took slightly less time to complete the requirements of the main study. This effect may be caused by the bias developed while exposed to 2D PCP. However, this data should be interpreted with caution. The participants did not have a clear cut-off point. For example, the study only finished once all the potential patterns were found as judged by the participant. There was also an age difference between the two groups; novice participants were in their twenties (P1, P2, P7) whereas experts were in their thirties (P3, P4, P6) or forties (P5). Furthermore, the experts had little or no familiarity with VR or the controller, which put them at a disadvantage compared with younger participants, such as P7, who reported extensive experience with this type of gamepad. This 
may have impacted their approach used during the study.

All the participants quickly became fluent in their chosen movement and manipulation strategies and did not report any comments or suggestions directly linked to these interaction techniques.

None of the participants generated more than a single scatter plot comprised of different dimensions even though they were informed about this possibility existed. Such behavior, however, may have been observed because only three criteria were present in the dataset. Hence, all of them could be mapped onto a single scatter plot. Moreover, the training phase also exposed participants to the methodology of exploring the data mainly through the outputs' scatter plot which was also used by Kipouros et al. [1] in their exploration of this dataset. However, some of the participants—P1, P2, and P6-reflected on the fact that having more than a single scatter plot may be confusing.

We observed that participants P4 and P6 chose to re-position the scatter plot closer to themselves, or the particular part of the main plot, instead of moving towards the scatter plot floating above the center of the IPCP plot. This behavior would most likely change if more data items would be visualized at once. Therefore, the movement would take considerably more time, and the perspective would render the distant selection of the elements at the scatter plot almost impossible.

Only participants P2 and P6 chose to rotate the scatter plot. However, we observed that for all participants the exact mapping of the dimensions to the 3D projection was not critical and this was also confirmed later in validation. The participants were selecting clusters on the scatter plot irrespective of the current rotation of the projection. One participant (P3) was constantly selecting the same, largest cluster, instead of exploring other groups or individual points.

\section{E. Design Principles}

From the technical constraints of what is achievable in VR, prior work [5], and literature review, as well as from expert feedback [1], and observations from our study we distilled six design principles:

D1-Immersive PCP visualization. The IPCP design should leverage the benefits bestowed by the immersive, spatial environment and remain both useful and compelling to the user. Prior work [23] has shown that standard 2D PCP may not benefit from translation into 3D. In fact, it can yield worse results due to, for instance, occlusion effects. Hence, the immersion bestowed by the VR environment should be considered as a key factor when designing a 3D PCP.

D2-Pattern candidate identification. The user must be able to identify candidates, i.e. a group of data items, as possible patterns within the dataset. This is the key functionality as the main purpose of the PCP visualization is aiding pattern identification within the given multidimensional dataset [13].

The next four design principles D3-D6 are derived from the first two principles D1-D2. Effective interaction, especially in the case of complex, multidimensional visualization in 3D space, requires the possibility of swift dataset inspection D3 and exploration D6. In turn, these two factors influence how the patterns are compared D4 and stored D5 for future reference. Without such functionality, the user may not be able to gain an overview of the whole, or parts, of 
the visualization and effectively analyze the IPCP. This is due to the sheer size in terms of the number of data items and how much space such a visualization can occupy in a spatial VR environment. Further, as the patterns may overlap, the system has to provide the user with the means of comparing previously found patterns with new candidates.

D3-Pattern candidate inspection. The user has to be able to inspect candidates for potential patterns within the dataset to decide if the particular group of data items forms a pattern.

D4-Pattern candidate comparison. To avoid repeated selections of the same pattern, the user has to be able to effectively compare a group of data items that could potentially form a pattern but also be able to compare such a group with previously discovered patterns.

D5-Pattern storage. The possibility of saving and retrieving on-demand information about discovered patterns is necessary for the users to be able to differentiate between potential new candidates and previously discovered patterns.

D6-Visualization exploration. The system should support exploratory visualization by providing mechanisms for zooming and exploring individual visualization elements.

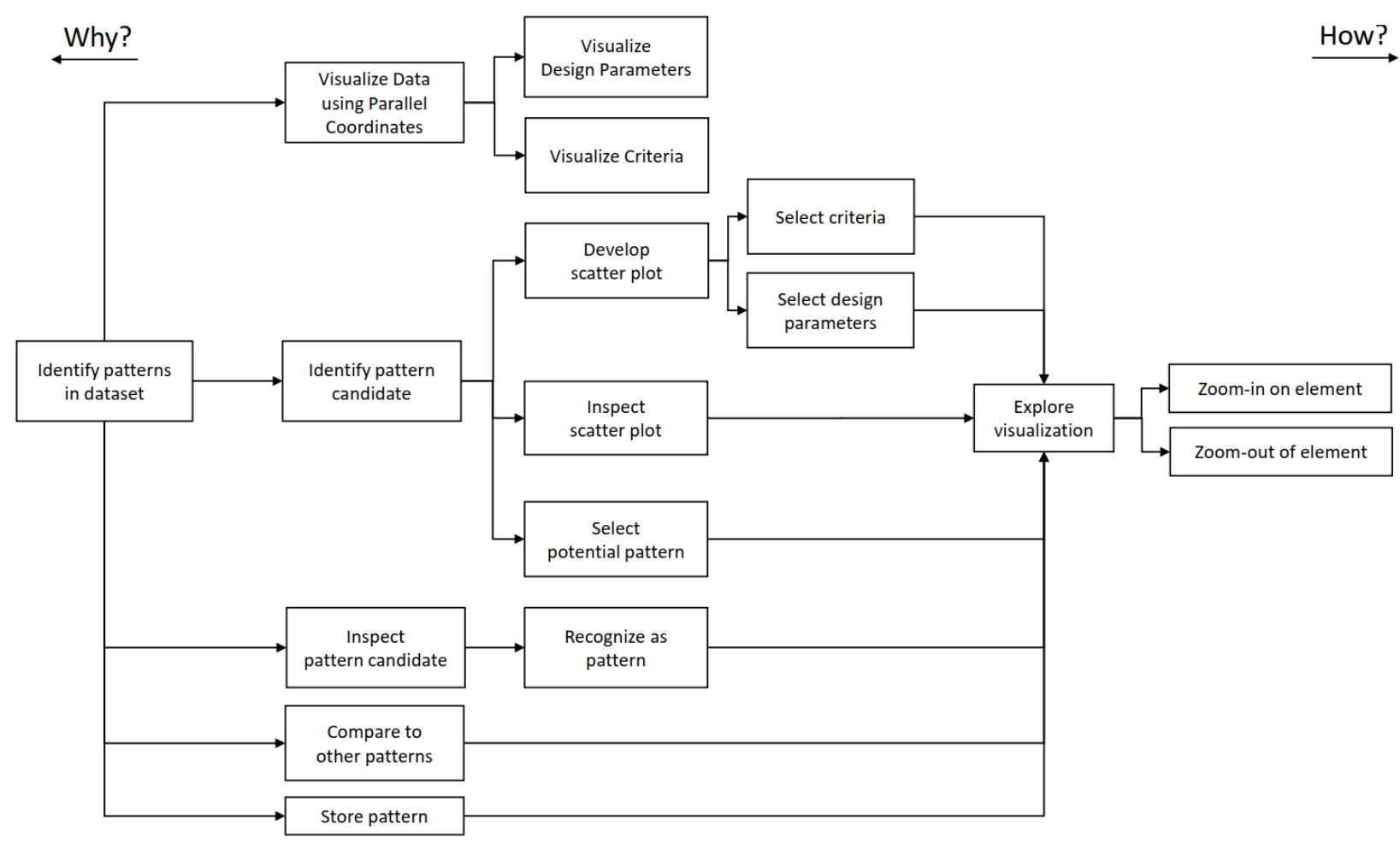

Fig. 7 The FAST diagram [33] used to reason about the requirements of IPCP. The horizontal axis shows abstraction level: higher-order functions to the left are gradually decomposed into lower-order functions to the right. The vertical axis shows order of execution. 


\section{F. Task Analysis}

We performed a task analysis by first identifying a suitable user interface workflow guided by the previously described user study. Each task is broken down into functions carried out either in series or in parallel. Thereafter, each function was translated into a function carrier (solution) by considering the requirements, technical constraints, and data from the user study. This analysis was carried out by first modeling the function structures with the help of the Function Analysis Systems Technique (FAST) [33] (see Fig. 7). Next, by using information gained through the formative user study in the form of observation and user comments and discussion, we have arrived with a list of six key tasks:

D1: Visualize data using IPCP. This task can be implemented in many variants in both 2D and 3D settings. For instance, one can use parallel planes [23, 26] to visualize the parallel axes. Instead, in our case, each data item is essentially a series of connected, interactive cubes spaced in equal intervals over the $X$-axis. Identically to 2D PCP, the $Y$-component denotes the value for each dimension. However, as we are spreading the points also in the third dimension, the cube's position in $Z$-axis signals the data item indexing with the first element starting with the $Z$-component equal to zero and progressing towards the positive direction of the $Z$-axis (see Fig. 2 or Fig. 3. As opposed to other 3D implementations, dropping the parallel planes limits the potential effects of occlusion and allows users to gain a birds-eye overview of the dataset by positioning themselves above the IPCP.

None of the participants reported confusion concerning the way the system visualized PCP. Moreover, all of them quickly understood that the interactive cubes indicate the numerical values in each dimension and that a group of line-connected cubes constitutes a single data item.

D2: Pattern identification. This task can be carried out in two ways: via exploration of the IPCP and visual inspection of a subset of selected data items, or with the scatter plot, as it was strongly suggested to the participants during the user study. However, we observed that the participants preferred a mixture of both approaches, that is, in many cases, a participant augmented a subset of data items selected with the help of the scatter plot by adding or removing selected data items individually from the PCP visualization.

D2*: Scatter plot generation. This task provides a feasible approach to pattern identification, especially when handling a large volume of data visualized with IPCP. The IPCP is augmented with the help of scatter plots [1, 24] generated from values in one, two, or three dimensions across all the data items. We gave the participants the ability to create up to five different 3D scatter plots. We also advised our participants to start by selecting the criteria to develop the scatter plot and see if they can use this aid to identify clusters of data points on the scatter plot to help them efficiently select a group of data items that can potentially form a pattern.

The scatter plots were augmented with additional functionality, such as the ability to apply rotation in $90^{\circ}$ about a chosen axis [6]-8]. In addition, they could be moved into any position in 3D space [6-8].

Based on the participants' comments we decided to contextualize the data points and scatter plot axes by providing textual labels, as seen in Fig. $5(\mathrm{a}-\mathrm{b})$. The labels contained information of which dimensions, that is, which $Y$-values, 
were mapped to which coordinates on the scatter plots. However, the two expert participants suggested a re-evaluation of this design, as they found the labels not to be very helpful.

If two or more values in a particular dimension are identical within the floating-point precision of their coordinates, they will be presented as two entirely overlapping cubes on the scatter plot. This effect would interfere with the ray-tracing used for gaze tracking as the system has no means to decide with which overlapping object the user wants to interact with. The analogous situation may also happen in the 2D PCP. In such a case, only a single point will be visible on the scatter plot, the selection of which will highlight multiple corresponding items on the IPCP as those points would be automatically clustered with each other. The simple clustering algorith (see Fig. 6) was nonetheless quite effective. The clustering method only considers the Euclidean distances between the cubes' (data points) centers, from which we estimate the level of overlap between the cubes. Certain restrictions, such as a difficulty of differentiating with which object users want to interact with if they are entirely overlapping, cannot be easily solved. We conjecture that different clustering algorithms for multidimensional data, such as one proposed by Wegman et al. [34] might be applied here. One way to minimize occlusion and cluttering effects in PCP is to use the density information of the data. This is shown in Heinrich et al. [35] and also in Artero et al. [36], where they proposed algorithms that use frequency and density information to decrease the overall number of data points that are shown to the user in PCP. Moreover, giving the user the possibility of zooming in and out of the cluster internal structure may prove to be very convenient in such situations.

D3: Inspection of the selected data items. This task is made possible through visual inspection of the selected items by either rendering not-selected points temporally invisible or by making a copy of the subset and placing it in a chosen part of 3D space. In both cases, participants usually first tried to gain an overview of the entire selection by positioning themselves (i.e., zooming out) in front of the subset and then compare and decided if particular data items constitute a pattern.

D4: Comparison of the new candidate to other patterns. This task is achievable by placing candidates near each other in 3D space and then visually inspecting possible overlaps. However, one of the participants mentioned that having some analytical aid could be helpful, especially if the data items are confounded by many dimensions. This participant also suggested using color-coding to provide additional information on how particular subsets potentially overlap with each other.

D5: Storage of the identified patterns. The system automatically logs a list of data items selected by the user into a file. Second, the sub-selection of data items can be placed and stored in any part of the 3D space throughout the entire duration of the visualization. However, as the IPCP extends along the $Z$-axis it may be difficult to compare points that are placed on the opposite sides from each other.

In addition, participants also remarked that a combination of both of these methods could potentially be even more beneficial. The logged data can be made re-callable, visualized on-demand to assist the user in understanding, for example, if, and to what extent, patterns, overlap between each other by additional color-coding or temporal shape 
change.

D6: Exploration of the visualization. The movement and manipulation methods allow the user to zoom-in or zoom-out on any visualization element, which, as reported by the participants, was easy to use with either Xbox Controller or Oculus Touch (see Fig. 4).

Based on the gathered qualitative data, all the participants swiftly understood the proposed movement and manipulation techniques. However, participants unfamiliar with the controller had problems remembering which button activated which action. This problem can be rectified by several strategies. First, the training can be extended. Second, the feature set can be narrowed. Third, the feature set can be initially limited and gradually expanded as the user gains proficiency in the interface. This third strategy is an example of a training wheel interface [37]. The ideal solution is probably a combination. In the short-term, extending training is preferable as PCP, even in its 2D version, demands intensive practice to gain fluency in their usage [32]. Another possible option would be to use a different VR headset (e.g., HTC Vive) that supports extended spatial tracking of the wearer. In such a case, instead of using a controller for movement and maneuvering the user can move around in the real world which would be instantly reflected in the VR environment. This would decrease the number of required interaction methods, although it would raise other issues, such as the increased complexity in tracking set-up and space demands for the system.

\section{Verification and Validation}

\section{A. Interface Verification}

We verified the usability of the system using the widely adopted Nielsen's heuristics [38, 39]:

1) Visibility of system status: The system constantly informs the user which visualization elements are in a selected state on both the IPCP (see Fig. 2 and Fig. 3 ) and on the scatter plots (see Fig. 5 and Fig. 6). The selected objects remain highlighted (in light green or orange) up to their deselection or when the user invoked visualization reset. This provides the user with continuous feedback about the state of all individual elements. Moreover, the system supports linking \& brushing interaction techniques by automatically mapping the current state of each of the visualization elements, i.e. scatter plots and the IPCP onto each other. Furthermore, if the user reaches the limit of possible duplicates, the system will disallow this operation and signal this to the user by playing a short sound.

2) Match between the system and the real-world: Both the 3D scatter plots and the cross-hair based gaze-tracking [58] should be familiar to the majority of users. The use of the cross-hair to represent the user's gaze point and the focus the user's attention on an object directly in front of where the user is looking at the moment was implemented based on a well-known real-world concept [9]. This, in turn, allows the system to optimize its resources to provided immediate feedback and response to the user's actions. 
3) User control and freedom: The user can reset [5- $[8]$ (see button $[R]$ in Fig. 44) the visualization to its original state which will preserve the copied data items (see Fig. 3). However, due to efficiency reasons, these duplicates will no longer support any kind of interaction after the system reboots and remain in "a freeze" state based on where they were originally copied. To further foster user control and freedom—before validation with the larger dataset - the system was extended to support the undo \& redo operations i.e. the last selection of data item on the IPCP or data point on the scatter plot can be undone or redone if necessary by the simultaneous press of two thumb-rest buttons (one on each of the controllers, see button $[H]$ in Fig. 4 ].

4) Consistency and standards: Despite the recent rapid development and advances in the research, VR technology is still in its early stage. As a consequence, the standards regarding the interaction techniques, visualization guidelines, or the overall design principles are not yet fully understood. We were trying to be consistent with well-known concepts, thus, for instance, the selection of the object requires a double-tap of the gamepad button instead of a single click similar to what is necessary when selecting an object using a computer mouse.

5) Flexibility and efficiency of use: The "accelerator" provided for the benefit of advanced users was the possibility to generate multiple scatter plots. Each of them could map up to three dimensions from the IPCP. Moreover, some users possessed previous experience with the Xbox Controllers (see Fig.44, and therefore required substantially less time to acquaint themselves with the interaction techniques.

6) Error prevention: The most common "slip-type" of an error related to user failing to focus his or her gaze on a chosen point is easily fixable with maneuvering techniques which allow the user to zoom-in the object by just moving towards it. The scatter plots automatically deselect the rotation selector when another one is being selected by the user [5-[8]. This prevents the system from confusion about which axis the scatter plot should be rotated. Moreover, the system will omit visualization of the data items on the PCP and the scatter plots if there is a gap in values at the input dataset, hence preventing the user from working with broken or incomplete data.

7) Help users recognize, diagnose, and recover from errors: The system uses the audio feedback to inform the user if no more duplicates of the selected data items can be generated immediately after such action was performed.

8) Recognition rather than recall: The linking \& brushing interaction techniques help to minimize the user's memory load by providing constant feedback about which operations have been previously executed by the user. Moreover, the user can easily duplicate a group of data items for further inspection and comparison with other such groups. This feature can be used, for instance, to keep track of which group of points were previously recognized by the user as a pattern.

9) Aesthetic and minimalist design: The systems keep the information presented to the user to a minimum. Only selected elements are highlighted (see selected: data items on Fig. 2 movement selector on Fig. 5.a) and cluster on Fig.6.(c)). Moreover, the gazed-locked textbox is only shown once the user's gaze is hovering over a data point on the scatter plot and automatically disappear otherwise. Thus, minimizing the possible collateral cluttering 
and occlusion effects simultaneously providing additional information of which dimensions were used to map into this points' rearrangement on the particular scatter plot.

10) Help and documentation: As it is hard to find users experienced in both the PCP and VR the participants were trained in how to read the PCP and how to interact with our system through the gaze-tracking and the Xbox controller (P1-P7) or the Oculus Touch (P8-P9).

\section{B. Validation}

It is very challenging to meaningfully validate visual analytics tools in short-term lab-based studies. Traditional human-computer interaction (HCI) evaluation methods, such as A/B testing, rely on the experimenter's ability to explicitly control all controllable and uncontrollable parameters of the system and the task as much as possible. However, in visual analytics, this parameter space is very large. For example, the data density, the nature of the task, characteristics of the dataset used in the test, and the experience and motivation of the participants, are all variables that are likely to affect the outcome of an A/B test. In addition, easy-to-measure dependent variables, such as task time, are only partially relevant as one of the primary objectives of a visual analytics tool is to provide additional insight rather than enabling users to make decisions faster.

As a consequence, we validated the system design qualitatively in two independent user studies. We recruited two pairs of different surrogate expert users to participate in each study. These participants are referred to as P8 and P9 in the case of the study involving the same dataset as in the previous study (DS1) and P10 and P11 with the new, larger, more complex dataset (DS2). All four volunteers were also pre-screened with the complete version of the Ishihara's color deficiency test [30].

\section{Part I: 54 Data Items with 29 Dimensions per Element (DS1)}

Table 2 Group of indexes of data points identified as patterns by the participants P8 and P9. The two bottom rows contain the two patterns that were identified by Kipouros et al. [1] as can be seen in Fig. [1] in blue and red respectively. Here we report only the participants' best attempts to identify the two patterns.

\begin{tabular}{|c|c|c|}
\hline Pattern candidate & P8 & P9 \\
\hline 1 & $33,34,35,36,38,40,41,42,43,46,49,52$ & $8,9,10,12,14,15,16,17,18,27,28$ \\
\hline 2 & $8,9,10,11,12,13,14,15,16,17$, & $8,9,10,11,12,13,14,15,16,17,18,23,24,25$, \\
$26,27,28,29,30$ \\
\hline Pattern A & $18,21,22,23,24,25,26,27,28,30$ & \\
\hline Pattern B & $32,33,34,35,36,38,39,40,41,42,43$ & \\
\hline
\end{tabular}

To validate the system design, we used the same three-part procedure and dataset DS1 (see Fig. 2) as in the first study reported earlier in this paper. However, based on the previous participants' feedback, we skipped parts of the presentations related to having multiple 3D scatter plots, although this possibility was not removed from the IPCP. As such, the training phase was truncated. Overall, the results revealed that participant P8 managed to find the majority of the elements in both patterns, $A$ with a $9 / 11$ completion ratio and $B$ with an $18 / 19$ completion ratio, whereas the second 
participant P9 correctly identified only the entire pattern $B$ with a $19 / 19$ completion ratio.

\section{Part II: 166 Data Items with 39 Dimensions per Element (DS2)}

Table 3 Group of indexes of data points identified as patterns by the participants. The four bottom rows contain the four patterns that were identified by domain experts [4]. In the case of P11 we excluded repeated pattern selections.

\begin{tabular}{|c|c|c|}
\hline Pattern candidate & P10 & P11 \\
\hline 1 & $93,96,102,103,105,119,120$ & $44,46,49,56$ \\
\hline 2 & $5,9,12,25$ & $92,95,98,106$ \\
\hline 3 & $13,15,17,19,20,21,23,29,30,31,42,43$ & $3,12,14,25,27$ \\
\hline 4 & $2,3,4,5,8,9,12,14,18,25,27$ & $34,37,38$ \\
\hline 5 & $44,46,49,56,59$ & $1,10,114,116,117,121$ \\
\hline 6 & $90,92,95,98,104,106$ & $2,3,7,18$ \\
\hline 7 & - & \\
\hline Pattern C & $44,45,46,48,49,51,56,59$ & \\
\hline Pattern D & $57,61,62,63,64$ & \\
\hline Pattern E & $13,15,17,21,29,30,31,34,35,37,38,42,43$ & \\
\hline Pattern F & $2,3,4,5,8,9,12,14,18,22,25,27$ & \\
\hline
\end{tabular}

After extending the system with additional capabilities, such as the support of basic undo \& redo operations we reran our validation study with another two domain-experts (hereafter referred to as P10-P11). We also used the Oculus Touch instead of the Xbox gamepad controller to facilitate the interaction (see Fig. (4).

We further investigated the feasibility of using IPCP for pattern identification in a substantially larger and more complex dataset DS2. The user study was carried out over a new dataset containing 166 data items with 39 dimensions per element containing 4 patterns of interest identified by domain experts (see Fig. 3). This dataset describes the results of the optimization study of S-duct design (see Fig. 8), as presented in D‘Ambros et al. [4].

In addition, the near-misses in terms of selection were the main observable issues with our selection technique. To support the user's selection task, one can use a form of target acquisition aid, that in our case, has been loosely inspired by the Bubble Cursor proposed by Grossman et al. [40]. We leveraged the features natively supported by the Unity game engine by on-the-fly manipulation of the size of the sphere collider [41] component attached to the interactive objects (cubes) in the IPCP. The size of the collider, which acts as a target for the raytracer [9], is dynamically adjusted based on the size of cross-hair (see the orange circle in Fig. 3) which in turn is dynamically scaled with respect to the user's distance from the target objects [9]. Initially, we set the collider's radius as $\sqrt{3} / 2 \approx 0.866$ to encompass the entire cube in the sphere. The further away the user is from the object, the larger the collider becomes.

P10 found $5 / 8$ of pattern $C, 9 / 13$ of pattern $E$, and almost entire pattern $F: 11 / 12$. P11 found 4/8 of pattern $C, 4 / 13$ of pattern $E$, and $9 / 12$ of pattern $F$ in his best attempt. However, none of the two participants found the pattern $D$. They did, however, identify other potential patterns that were not initially found by the original researchers, which is a concrete demonstration of the utility of the system in enabling users to explore and identify previously unknown patterns in the dataset. Interestingly, some overlapping results were reported by both participants, as can be seen in Table 3 . 
namely pattern candidate 6 for P10 and pattern candidate 2 for P11. Moreover, both participants identified additional groups of points that in their opinion constituted patterns not originally identified by the experts in D“Ambros et al. [4]. As mentioned before, in the case of visual analytics, we aim to provide additional insight into the dataset, hence we were interested in the new candidates for potential patterns found by the two participants (P10 and P11). To do this, we requested a domain-expert to re-analyze and comment on the new pattern candidates identified. After inspection and preliminary analysis, the expert concluded that indeed these are different patterns sharing some common characteristics with those identified previously and as such they are complementary. However, the domain expert commented that they are not as rich as the dominant four patterns previously identified, i.e. $C, D, E$, and $F$. Nonetheless, this tentatively indicates that the VR analytics system can empower users to detect new patterns.

\section{Design Decisions}

We summarize the final design decisions refined after we ran additional experimental studies with domain experts over two separate datasets (see Fig. 2and Fig. 3) varying in both the number of data items and dimensions per data item as well. Moreover, based on the participants' observed behavior and their comments we also identified a number of potential improvements.

D1-Immersive PCP visualization. We have developed IPCP as a software system running within the Unity [41] game engine. Our approach represents a 3D version of regular 2D PCP but with values of each dimension denoted as volumetric, interactive objects, that is, cubes spread in the $Z$-axis instead of using parallel planes as regular 3D PCP usually does. However, this way of presenting data has its own challenges. For instance, to clearly see the distant objects with respect to the user's current position, it was necessary to provide adequate means of close inspection of such regions. Here, we give the user the possibility to freely move and maneuver in the 3D space using the controllers (Xbox gamepad in first and Oculus Touches in the following two observational studies). Such approach has additional advantages, especially when we coupled the IPCP visualization with the CAD models of the respective designs (see Fig. 8) which now can be inspected not only from outside but also from within as well (see Fig. 8(c)). Another possibility is to give the user means of manipulating the entire PCP visualization with the user's hands as shown in Fig. 10,13

D2-Pattern candidate identification. In the context of PCP, the pattern is understood as group of data items sharing similar To identify and highlight a group of data items that can potentially form a pattern, the user either selects data items one-by-one or by generating a 3D scatter plot from up to three dimensions. This data is automatically clustered based on the distance between the data points (see Fig. 6) and the selection of such data points belonging to a cluster simultaneously highlights points belonging to this cluster on the scatter plot and the IPCP alike. The larger the data set and subsequent IPCP visualization, the more the scatter plots seem to aid the user in the task of finding patterns. Here, we noted that the actual orientation of the scatter plot's local axes is not as important as providing the user with a clear way of distinguishing which points on the scatter plot potentially form a cluster. We observed 
that even a simple clustering algorithm, however relying on the user's intuitive feel of the grouping of volumetric points placed in close vicinity can be surprisingly effective. Hence, potential improvements could be threefold. First, other clustering algorithms can be studied here such us Ordering Points to Identify Cluster Structure (OPTICS) [42] or Density-Based Spatial Clustering of Applications with Noise (DBSCAN) [43] as they can, by design, handle noisy data, e.g. containing almost constant dimensional values across all the data items, such as the one used in our experiments. Second, different methods of the 3D scatter plot manipulation can be used. For instance, instead of rotating the scatter plot along with one of the three main axes, it could be rotated about any vector selected by the user (see Fig. 11]. These would potentially help the user to swiftly obtain an overview of the entire data present at the scatter plot. Third, to avoid repeated selection of the same cluster, and subsequent repetition of the same group of data items on the IPCP, we could additionally color-code or otherwise previously selected and inspected clusters. Furthermore, additional information could be given in a form of textual labels attached to the data points instead of current axes mapping, such as the scatter plot's coordinates or a number of points within the selected cluster which could be more informative to the users.

D3-Pattern candidate inspection. To inspect the pattern candidates, the user can temporally disable the unselected data items of the IPCP and use the manipulation and movement capabilities in the 3D space to verify if this particular group of data items forms a pattern. Moreover, if a particular data item is upon inspection deemed by the user to not be part of this pattern, it can be easily deselected as well. This is especially useful the more data items are contained within the dataset. As the data items will be spread in the $Z$-axis (see Fig. 2] and Fig. 3 ) the potential occlusion effects are more persistent with the growing number of data items. Here, the ability to toggle between the view with all or only currently selected data items has proven to be very useful.

D4-Pattern candidate comparison. To compare the currently selected group of data items with previously recognized patterns, the user can make a copy of these data items, i.e., the pattern candidate, and place them in a part of 3D space where the other patterns have been stored. The user may then visually compare them to decide if a new pattern has been found. Here, the system should provide the user with the "snapping" mechanism that would automatically align the subsets of the data items that the user wants to compare with each other. To some extent, the participants were trying intuitively to obtain a similar effect by placing the duplicates of previously found patterns next to each other in the $3 \mathrm{D}$ space.

D5-Pattern storage. Previously discovered patterns of data items can be stored freely in any location within 3D space. Here, even though we could keep the "frozen" data items as non-interactive for optimization reasons, we could color-code the data items overlapping across the previously found patterns and the new group of points to inform the user to what extent they share the same subset of data.

D6-Visualization exploration. Exploration of the entire visualization with all of its elements is made possible through the use of gaze-tracking, head-tracking, and movement assisted by the controllers. Here, we observed that all our participants swiftly gain fluency in how to move around the virtual space. We can also leverage the tracking 


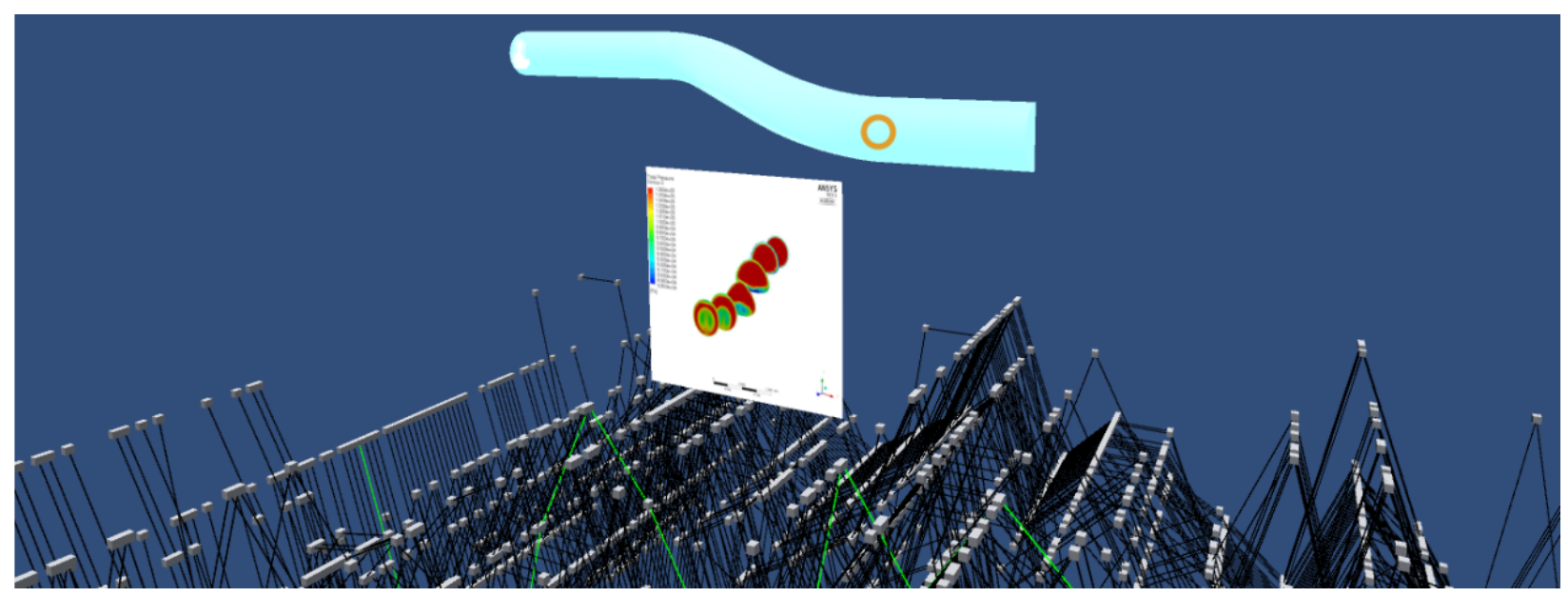

Fig. 8 After the selection of an item on IPCP, the user is shown additional information, for example, an S-duct CAD geometry and a 2D plot of the S-duct flow.

of the user in a real space, especially if we combine this with the bimanual manipulation of the PCP elements (see Fig. 10-13). However, in our case, the IPCP-pending on the input data size-can be potentially vastly extended in at least the $X Z$-plane parallel to the user actual ground-plane. As such, it could be unfeasible for the user to walk and see the entire visualization, hence new interaction and transportation methods would have to be included.

\section{Visualization Extensions}

\section{A. Extensions of the IPCP}

After analyzing both the qualitative and quantitative feedback together with the participants' comments and our own observations, we have decided to extend our system with a number of additional features. We have also decided to explore a completely new method of interacting with the system using hand tracking-based gestural input coupled with gaze-tracking (see Fig. 9). The changes were twofold.

First, we have equipped the system with the Computer-Aided Design (CAD) 3D models of the individual designs to allow the user to inspect and asses the associated geometries on-the-fly while selecting data items on the (see Fig. 8).

Second, instead of using hand-held controllers (see Fig. 44, this system iteration explores gaze-tracking coupled with hand-tracking supported by an additional sensor (see Fig. 9], namely the Leap Motion Sensor [29] attached to the VR head-mounted display to track and recognize the user's hand gestures.

Finally, we report the results of an interface verification of the system guided by Nielsen's heuristics [38, 39]. This allows us to identify the main usability bottlenecks and issues with respect to the new gesture-based interaction method. 


\section{Coupling IPCP with Computer-Aided Design (CAD) Geometries}

The system contains the visualizations of associated 3D models of the full 3D S-Duct geometries and 2D flow solutions, an example of which can be seen in Fig. 8 . In the future, we are also planning to give the users the possibility of overlaying the various S-duct geometries on top of each other to closely inspect and identify potential nuances in the difference between the shapes as demonstrated in the AeroVR system presented in Tadeja et al. [7, 8] and upon which this visualization is built. This allows the users to promptly verify their pattern selections and compare between the S-duct shapes associated with selected data items. This extension also fosters the connectivity with commercial software and simulation packages that are widely used in the industry.

\section{Hand-Tracking and Bimanual IPCP Manipulation}

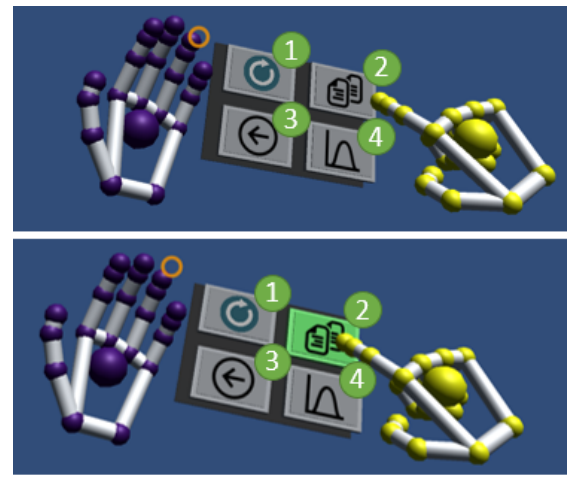

(a)

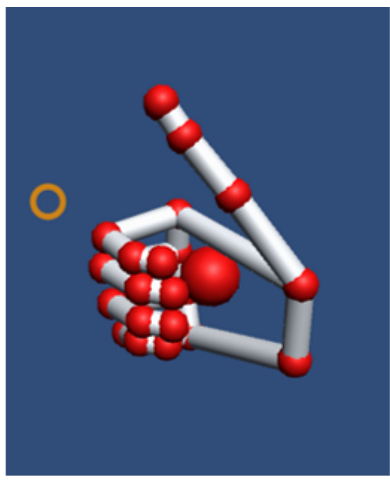

(b)

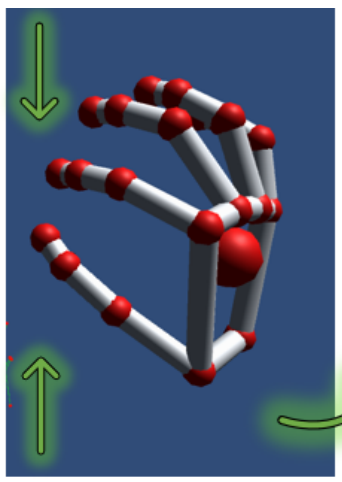

(c)

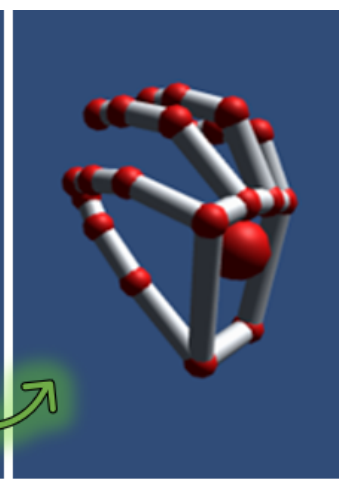

(d)

Fig. 9 The recognized gestures are (a) the left-hand menu; (b) the thumbs-up gesture; and the pinch gesture before (c); and after execution (d). Icons by Icons8 (https://icons8. com).

The user can manipulate all the visualization's elements with the help of a new, bimanual interaction technique and a range of gestures (see Fig. 9) without the need of using hand-held controllers (see Fig. 4). This was achieved through a mixture of gaze-tracking and hand-gesture recognition. The left-hand menu (see Fig. 9 (a)) recalls the hand-attached menu with all the available options: (1) [REST ART] resets the visualization to default settings; (2) [DUPLICATE] duplicates the selected data items at the location in 3D space the user is currently gazing whilst pressing the button; (3) $[U N D O]$ reverts the results of any manipulation of either the IPCP or scatter plot depending on which of the two is selected while pressing the button; and (4) $[T O G G L E]$ toggles between the view with only visible selected data items in the IPCP. The thumbs-up gesture (see Fig. 9 b)) is used to release selection of an interactive object. The pinch gesture (see Fig. 9 (c-d)) executed with the index finger and thumb allows the user to select and control interactive objects. After gazing on an interactive object the user can activate or deactivate control over this object by performing the pinch gesture.

In case of the IPCP main plot, the users can select (see Fig. 10) either a single data item using the single-pinch gesture (see Fig.10(b)) or the entire dimension across all data items using the double-pinch gesture (see Fig. 10 (c)). In 


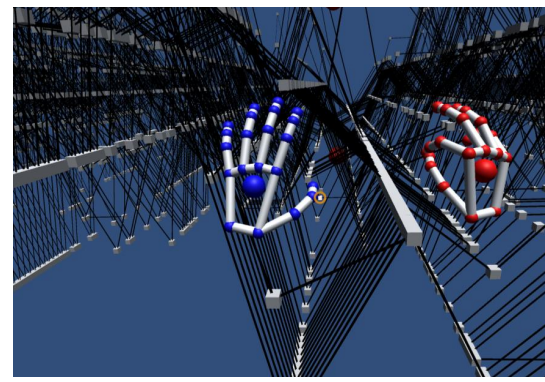

(a)

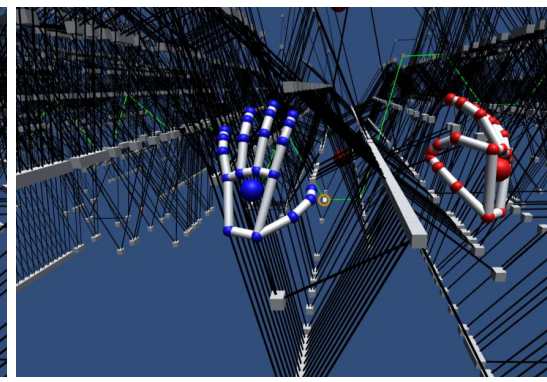

(b)

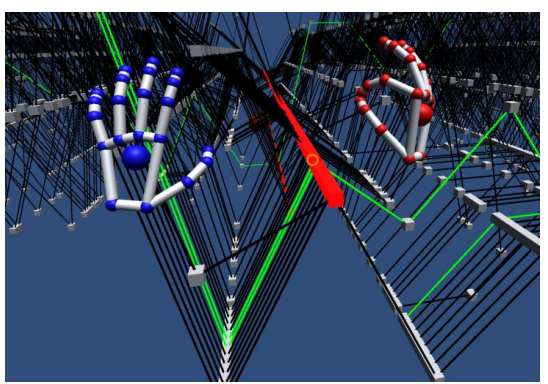

(c)

Fig. 10 Data item selection: (a) gazing with a cross-hair; (b) selecting data items with single-pinch gesture; (c) or selecting the entire dimension with double-pinch gesture.

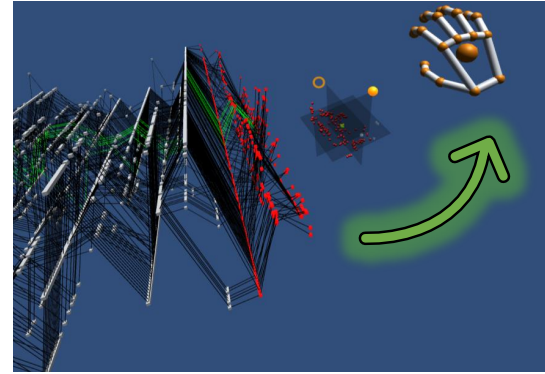

(a)

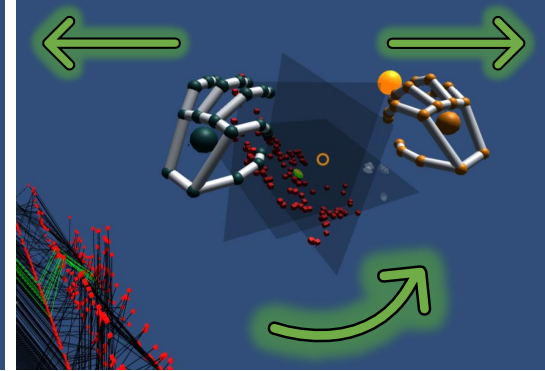

(b)

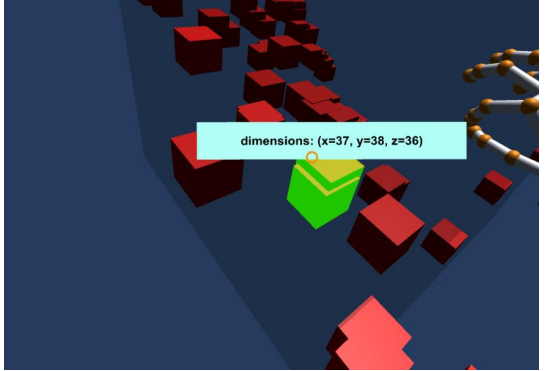

(c)

Fig. 11 (a) the 3D scatter plot [7, 8]; (b) bimanually enlarging and simultaneously rotating the scatter plot; (c) gazing over a selected cluster and recalling a pop-up textbox.

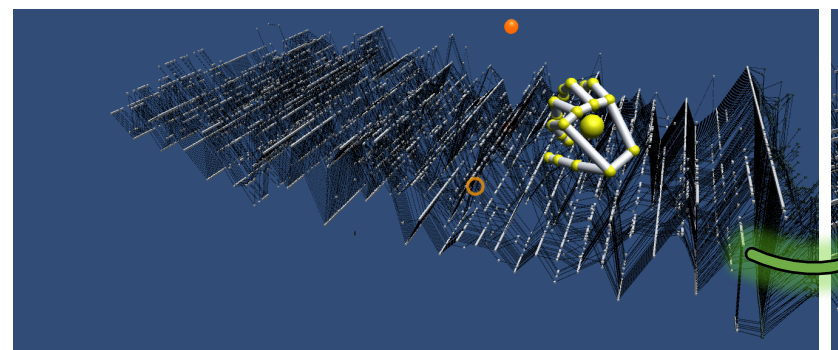

(a)

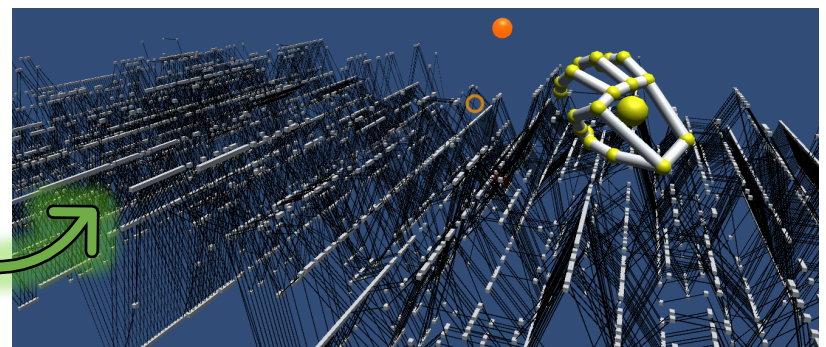

(b)

Fig. 12 The IPCP movement procedure: (a) gazing over and selecting the IPCP's selector with a double-pinch gesture; and (b) moving the IPCP with either hand.

addition, there are two interactive movement selectors situated above and below the main plot.These selectors have a form of spherical markers whose, similarly to scatter plots, color-coding is switch to orange after selection as seen in Fig. 12 or in Fig.13 After activating one of these selectors, users can manipulate the whole IPCP by:

(i) Rotating the 3D scatter plot (see Fig. 11): (a) shows the scatter plot generated out of three criteria dimensions of the DS2 dataset (selected in red); (b) illustrates the user enlarging and simultaneously rotating the scatter plots using both hands; and (c) depicts the user gazing over a selected cluster of data points in the enlarged scatter plot, which results in a text box with additional information. 


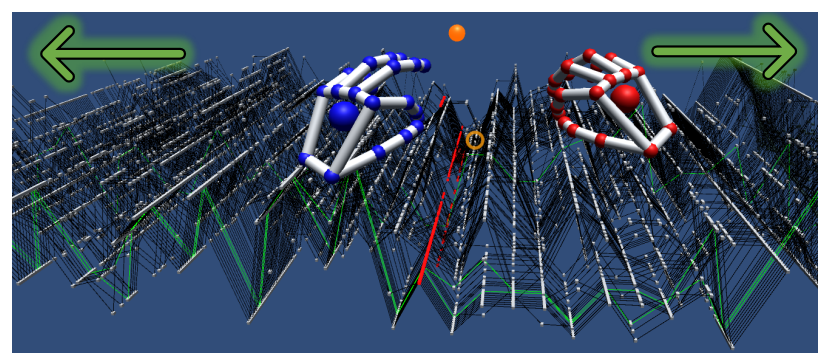

(a)

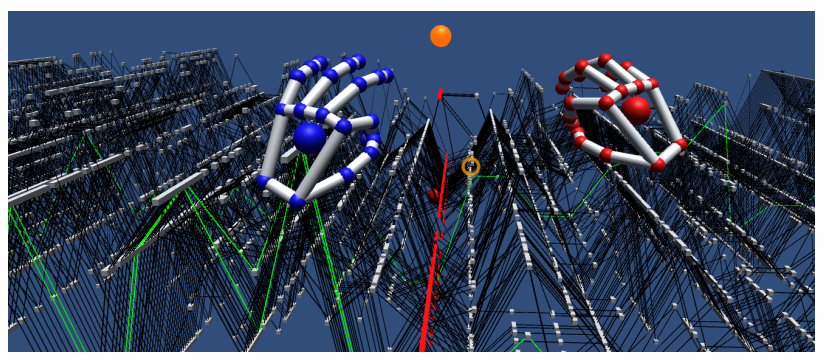

(b)

Fig. 13 The IPCP scaling procedure: after selecting the IPCP the IPCP is scaled by the user moving their hands further apart or closer together (a-b).

(ii) Moving the IPCP main plot (see Fig. 12): (a) after gazing at the IPCP's selector (red sphere) and selecting it with the double-pinch gesture the user can (b) move the IPCP with either hand.

(iii) Scaling the IPCP main plot (see Fig. 13): after selecting the IPCP's selector (orange sphere), and articulating a pinch gesture with both hands, the user can scale the IPCP by moving their hands further apart or closer together (a-b).

The 3D scatter plot can be generated out of a maximum of three dimensions selected on the IPCP, and it can be manipulated similarly to the main IPCP (see Fig. 11). Moreover, could allow the user to select a group of points that constitute a cluster based on one of the selected clustering algorithms such as mentioned earlier OPTICS [42] or DBSCAN [43]. A selection of the algorithms can be attached to the left-hand pop-up menu in place of the empty buttons shown in Fig. 9 a). Any other actions can be attached to those buttons as well. For instance, we could give the user possibility of attaching actions facilitated with the execution of the user-indicated external script or another program. Thus, vastly enhancing the IPCP's capabilities and simultaneously rendering IPCP more suited to the user's individual needs and wants.

\section{B. Verification of the Extended Interface}

The usefulness of basic IPCP and 3D scatter plot designs were verified and evaluated previously with the user studies in which the domain-experts were able to not only rediscover previously found patterns in the data but also, to a limited extent, to discover new ones. Here, the updated version of IPCP was equipped with the new gesture-based interface. As such, the main goal here is to verify the impact of this new, bimanual interaction technique have on the interface and to reason about the potential benefits brought with it to the user. To achieve these we apply the formative evaluation method, i.e., we assess the interface's usability with the help of Nielsen's heuristics [38, 39].

Visibility of system status. The user is continuously informed about the current selection of data items at the IPCP, data points on the 3D scatter plots (see Fig. 10), activation of objects' selectors (see Fig. 11), and buttons selection (see Fig. 9 (a)). This is achieved through automatic color-coding and highlighting.

Match between system and the real world. The interface provides the user with the possibility to use gestural-input 
coupled with the gaze-tracking to select, manipulate, and interact with the main visualization elements the same way as the user would in the non-virtual world. These include the possibility to (i) rotate (see Fig. 11), (ii) move (see Fig. 12), or (iii) re-scale the size (see Fig. 13) of either the main IPCP plot, or the 3D scatter plot alike.

User control and freedom. The system features both [UNDO] and [RESTART] options (see Fig. 9 (a)). The former allows the user to undo the last executed manipulation of either the 3D scatter plot or the IPCP. These operations will not result in the loss of the previously made selections of data items and/or data points. The restart (see Fig. 97a)) will cause the entire visualization to be restored to its defaults.

Consistency and standards. In the case of the interactive objects' selectors, the same convention is followed i.e. both the scatter plot and IPCP main plot has the selectors in a form of large, spherical markers that have to be selected with the double-pinch gesture while the user is gazing over them.

Error prevention. The execution of the two operations that may cause irreversible effects on the current state of the visualization, i.e. the $[U N D O]$ and $[R E S T A R T]$ require the double-press of a button to be executed instead of a single-press required for the execution of the remaining options.

Recognition rather than recall. The user can execute the palms-up gesture which instantaneously recalls the left-hand pop-up menu with all the available options (see Fig.9.a)).

Aesthetic and minimalist design. Only the main IPCP is initially visible to the user unless the user decides to make copies of the groups of selected data items or generate the scatter plot. However, if no dimensions on the IPCP are selected, the scatter plot will not be visible.

\section{Discussion}

The IPCP tool automatically provides a rudimentary form of collaborative design environment. The first-person view of the headset wearer can also be simultaneously streamed on the PC display as well. As a consequence, multiple designers can look at the steps undertaken by the user and easily collaborate having a verbal dialogue between themselves and the person using the headset. This capability was observed by the authors while working on the design and verification of the IPCP tool presented in this paper. The advantages of such a setup will be further studied in a series of more controlled user studies.

\section{A. Limitations}

The evaluation with a group of surrogate expert users, P8-P9 for the smaller (DS1) and P10-P11 for the larger dataset (DS2), revealed that users were indeed capable of identifying patterns in both datasets. However, while this evaluation demonstrates the efficacy of the system and serves to provide some evidence to consider the potential merits of 3D PCP, we advise caution against over-interpreting these results given the qualitative aspect of the findings. The sample size is very small and, as a consequence, the findings are highly sensitive to the individual participants' general 
ability to use novel immersive technology to analyze a demanding real-world multidimensional dataset.

A previous study conducted by Johansson et al. [23] indicated that 3D PCP was, in fact, worse than a conventional 2D version in both the metrics (i.e., response times and error rates). However, the study was conducted using a desktop computer connected to an external display instead of an immersive, VR environment, and thus it is unclear whether the findings generalize to a truly immersive environment. However, such a study is intrinsically limited in terms of the many study parameters that have to be arbitrarily set in order to be able to achieve an $\mathrm{A} / \mathrm{B}$ comparison. Examples of such study parameters include data density and volume, saliency of data items, field of view, the user's sense of presence, situational awareness of a 3D world etc. The objective in this paper is to explore whether VR is feasible for PCP. Our results demonstrate that PCP is indeed viable for VR analytics and thus encourage and motivate further design exploration. However, as PCP in VR is still in its infancy, it is important to avoid a premature 2D vs. VR comparison until an effective PCP in VR design has fully emerged.

\section{B. Future Work}

Future work includes conducting statistically robust quantitative studies that are able to tease out the merits and flaws of immersive PCP visualization compared with status-quo 2D PCP visualizations. However, such studies are difficult to carry out due to the many controllable and uncontrollable parameters of the system and the experimental task. This makes the design of a robust and meaningful experimental design highly challenging. Prior work has reflected about this problem, both in general for evaluating visualization tools [44, 45] and specifically for the problem of comparing $2 \mathrm{D}$ and $3 \mathrm{D}$ visualizations [46].

In the near future we believe a more fruitful approach is to iteratively evolve a highly promising PCP in VR approach by following the model of this paper of combining design with qualitative insights gathered from domain experts. Once such a design has fully matured it will become more meaningful to carry out large-scale A/B comparisons capable of teasing out the likely nuances differences between PCP in 2D or VR.

We believe a promising area of future work is to further refine the system design by evolving the task model and the underpinning functions and revisit the process of translating functions to solutions. This can be achieved by identifying controllable and uncontrollable parameters for each overall function necessary to carry out a task and study the effects of modifying these parameters in isolation.

For example, the 3D immersive environment opens up a plethora of methods for selecting data points using input techniques such as head-tracking, gaze-tracking, touch controllers, game controllers, or direct manipulation mechanics. At the same time, selection feedback can be provided using a variety of output techniques, such as color-coding, shape modification, sound, haptic feedback, or a combination.

Further, the data analytics toolkit can be extended with a range of new features, including explainable AI methods such as LIME [47], SHAP [48] or Anchors [49] to allow the user to query more complex questions about the data, 
such as, "Why were these points classified as noise and not included among any of the identified clusters?". Another interesting research direction is to investigate coupling the system with a voice interface.

Our system implementation can aid such future explorations of how to make informed decisions on, for instance, which combination of inputs and outputs to choose to arrive at an effective operating point for immersive data analysis and thereby assist designers in gaining a more complete understanding of the trade-offs and their effects that are inherent in such design decisions. We believe such technological capability should be harmonized with state-of-the-art interactive computational engineering design methodologies [50] and thereby open up a new generation of engineering design software environments and systems.

\section{Conclusions}

The qualitative results in this paper reveal that immersing the user within PCP in a VR environment is, at the very least, complementary and may provide ways to enhance the user's ability to identify patterns. Thus immersive 3D PCP can potentially be considered as a promising way of visualizing high-dimensional data. Most of the participants throughout our qualitative studies managed to identify the same patterns as recognized as interesting in Kipouros et al. [1], which can be regarded as the reference standard. The two participants working with a substantially larger dataset in terms of its complexity and size-the number of dimensions per data item and the total number of data items — discovered similar patterns as those given in D`Ambros et al. [4] and identified additional candidates for potential patterns. This demonstrates the potential for immersive VR in engineering design processes.

The main contribution of this paper is the six design principles that we distilled based on the technical constraints of what is achievable in VR, prior work [5], literature review, expert feedback [1], and from observations from our study.

The second strand of contribution is the task analysis carried out at the functional level and the translation of these functions into solutions that allowed the system to be realized. This scaffolding structure is useful in framing the findings from the preliminary qualitative user study in such a way that the outcomes of the user study could directly shape the refinement of the initial design.

Further, we gradually updated the hardware interface for the interaction between the user and the IPCP. We switched from an Xbox gamepad to Oculus Touch controllers specifically designed for VR (see Fig. 4) and we replaced the controller-based interface with gestural input coupled with gaze-tracking (see Fig. 97. This enabled the user to manipulate the IPCP plot and 3D scatter plot in a similar manner to real-life objects.

To better inform the user's decision making and analysis of the dataset, we coupled the individual data items with additional information. These supplementary data can, among other things, include the 3D geometries, such as the S-duct designs for DS2 or the 2D plots of the flow going through the duct as shown in Fig. 8

In summary, the results in this paper serve as a promising indication of that immersive parallel coordinate plots may enhance decision making in complex design engineering processes, which are intrinsically reliant on the user being able 
to analyze and reason about multidimensional data.

\section{Funding Sources}

The work of Sławomir K. Tadeja was supported by the Cambridge European and Trinity Hall and the EPSRC (1788814) scholarships, whereas the work of Yupu Lu was supported by the Tsinghua Academic Fund for Undergraduate Overseas Studies and the Fund from Tsien Excellence in Engineering Program.

\section{Acknowledgments}

The authors would also like to thank Davide Dal Magro for providing us with the S-Duct CAD geometry and associated flow plots and to John Dudley and Michał Drewniok for proof reading the manuscript. We also appreciate suggestions from Szymon Bobek in regard to the selection of potential clustering algorithms and help of Przemysław Stachura in generating figures.

\section{References}

[1] Kipouros, T., Inselberg, A., Parks, G. T., and Savill, M., "Parallel Coordinates in Computational Engineering Design,” AIAA Multidisciplinary Design Optimization Specialists, Vol. 1750, 2013. https://doi.org/10.2514/6.2013-1750.

[2] Kipouros, T., Jaeggi, D., Dawes, W., Parks, G., Savill, M., and Clarkson, P., "Bi-Objective Design Optimization for Axial Compressors Using Tabu Search,” AIAA Journal, Vol. 46, No. 3, 2008, pp. 701-711.

[3] Kipouros, T., Jaeggi, D., Dawes, W., Parks, G., Savill, M., and Clarkson, P., "Insight Into High-Quality Aerodynamic Design Spaces through Multi-Objective Optimization,” CMES: Computer Modeling in Engineering and Sciences, Vol. 37, No. 1, 2008, pp. $1-44$.

[4] D’Ambros, A., Kipouros, T., Zachos, P., Savill, M., and Benini, E., “Computational Design Optimization for S-Ducts,” Designs, Vol. 2, No. 36, 2018, pp. 1-21. https://doi.org/10.3390/designs2040036

[5] Tadeja, S. K., Kipouros, T., and Kristensson, P. O., "Exploring Parallel Coordinates in Virtual Reality," Extended Abstracts of the CHI Conference on Human Factors in Computing Systems Extended Abstracts (CHI EA'19), Glasgow, Scotland UK, 2019. https://doi.org/10.1145/3290607.3313068

[6] Tadeja, S. K., Kipouros, T., and Kristensson, P. O., "IPCP: Immersive Parallel Coordinates Plots for Engineering Design Processes," Proceedings of AIAA SciTech Forum and Exposition, 6 - 10 Jan 2020, Orlando, Florida, USA, 2020. https: //doi.org/10.2514/6.2020-0324

[7] Tadeja, S. K., Seshadri, P., and Kristensson, P. O., "Exploring Aerospace Design in Virtual Reality with Dimension Reduction," Proceedings of AIAA SciTech Forum and Exposition, 7 - 11 Jan 2019, San Diego, California, USA, 2019. https://doi.org/10.2514/6.2019-2206 
[8] Tadeja, S. K., Seshadri, P., and Kristensson, P. O., “AeroVR: Immersive Visualization System for Aerospace Design,” https://arxiv.org/abs/1910.09800, Oct. 2019 (under review). URL https://arxiv.org/abs/1910.09800

[9] Unity3D Game Engine, "Unity VR Samples pack," https://assetstore.unity.com/packages/essentials/tutorial-projects/vr-samples51519. Last accessed: November 2018.

[10] Oculus VR, “Oculus Utilities for Unity 5,” https://developer.oculus.com, Last accessed: November 2018.

[11] Greyman studios S.L., “Off Screen Indicator,” https://assetstore.unity.com/packages/tools/gui/off-screen-indicator-57062 Last accessed: July 2017.

[12] Inselberg, A., “The plane with parallel coordinates," The Visual Computer, Vol. 1, No. 2, 1985, pp. 69-91. https://doi.org/10. 1007/BF01898350

[13] Inselberg, A., Parallel Coordinates: Visual Multidimensional Geometry and Its Applications, $1^{\text {st }}$ ed., Springer, New York, USA, 2009.

[14] Wegenkittl, R., Loffelmann, H., and Groller, E., "Visualizing the behaviour of higher dimensional dynamical systems," Proceedings. Visualization '97 (Cat. No. 97CB36155), 1997, pp. 119-125. https://doi.org/10.1109/VISUAL.1997.663867

[15] Gröller, E., Löffelmann, H., and Wegenkittl, R., "Visualization of Analytically Defined Dynamical Systems," Scientific Visualization Conference (dagstuhl'97), 1997, pp. 71-71.

[16] Streit, M., Ecker, R. C., Österreicher, K., Steiner, G. E., Bischof, H., Bangert, C., Kopp, T., and Rogojanu, R., “3D parallel coordinate systems-A new data visualization method in the context of microscopy-based multicolor tissue cytometry," Cytometry Part A, Vol. 69A, No. 7, 2006, pp. 601-611. https://doi.org/10.1002/cyto.a.20288

[17] Falkman, G., "Information visualisation in clinical Odontology: multidimensional analysis and interactive data exploration," Artificial Intelligence in Medicine, Vol. 22, No. 2, 2001, pp. 133-158. https://doi.org/10.1016/S0933-3657(00)00104-4

[18] Ribarsky, W., Ayers, E., Eble, J., and Mukherjea, S., "Glyphmaker: creating customized visualizations of complex data," Computer, Vol. 27, No. 7, 1994, pp. 57-64.

[19] Fanea, E., Carpendale, S., and Isenberg, T., "An interactive 3D integration of parallel coordinates and star glyphs,” IEEE Symposium on Information Visualization, 2005. INFOVIS 2005., 2005, pp. 149-156. https://doi.org/10.1109/INFVIS.2005. 1532141

[20] Johansson, J., Ljung, P., Jern, M., and Cooper, M., "Revealing Structure in Visualizations of Dense 2D and 3D Parallel Coordinates," Information Visualization, Vol. 5, No. 2, 2006, pp. 125-136. https://doi.org/10.1057/palgrave.ivs.9500117.

[21] Dang, T. N., Wilkinson, L., and Anand, A., "Stacking Graphic Elements to Avoid Over-Plotting," IEEE Transactions on Visualization and Computer Graphics, Vol. 16, No. 6, 2010, pp. 1044-1052. https://doi.org/10.1109/TVCG.2010.197. 
[22] Chang, C., Dwyer, T., and Marriott, K., “An Evaluation of Perceptually Complementary Views for Multivariate Data,” 2018 IEEE Pacific Visualization Symposium (PacificVis), 2018, pp. 195-204. https://doi.org/10.1109/PacificVis.2018.00033.

[23] Johansson, J., Forsell, C., and Cooper, M., "On the usability of three-dimensional display in parallel coordinates: Evaluating the efficiency of identifying two-dimensional relationships," Information Visualization, Vol. 13, No. 1, 2014, pp. $29-41$. https://doi.org/10.1177/1473871613477091

[24] Holten, D., and Wijk, J. J. V., "Evaluation of Cluster Identification Performance for Different PCP Variants," Computer Graphics Forum, Vol. 29, No. 3, 2010, pp. 793-802. https://doi.org/10.1111/j.1467-8659.2009.01666.x

[25] Cordeil, M., Cunningham, A., Dwyer, T., Thomas, B. H., and Marriott, K., "ImAxes: Immersive Axes As Embodied Affordances for Interactive Multivariate Data Visualisation," Proceedings of the 30th Annual ACM Symposium on User Interface Software and Technology, ACM, New York, NY, USA, 2017, pp. 71-83. https://doi.org/10.1145/3126594.3126613.

[26] Butscher, S., Hubenschmid, S., Müller, J., Fuchs, J., and Reiterer, H., "Clusters, Trends, and Outliers: How Immersive Technologies Can Facilitate the Collaborative Analysis of Multidimensional Data," Proceedings of the 2018 CHI Conference on Human Factors in Computing Systems, ACM, New York, NY, USA, 2018, pp. 90:1-90:12. https://doi.org/10.1145/3173574. 3173664

[27] Rosenbaum, R., Bottleson, J., Liu, Z., and Hamann, B., "Involve Me and I Will Understand!: Abstract Data Visualization in Immersive Environments," Proceedings of the 7th International Conference on Advances in Visual Computing - Volume Part I, Springer-Verlag, Berlin, Heidelberg, 2011, pp. 530-540.

[28] Ribarsky, W., Bolter, J., Op Den Bosch, A., and Van Teylingen, R., "Visualization and Analysis Using Virtual Reality," Computer Graphics and Applications, IEEE, Vol. 14, 1994, pp. 10-12. https://doi.org/10.1109/38.250911

[29] Leap Motion, “Leap Motion,” https://www.leapmotion.com Last accessed: September 2019.

[30] Ishihara, S., Ishihara's Tests for Colour Deficiency, $38^{\text {th }}$ ed., Kanehara Trading Inc, Tokyo, Japan, 2017.

[31] Kennedy, R. S., Lane, N. E., Berbaum, K. S., and Lilienthal, M. G., "Simulator Sickness Questionnaire: An Enhanced Method for Quantifying Simulator Sickness,” The International Journal of Aviation Psychology, Vol. 3, No. 3, 1993, pp. $203-220$. https://doi.org/10.1207/s15327108ijap0303_3

[32] Shneiderman, B., and Plaisant, C., Designing the User Interface: Strategies for Effective Human-Computer Interaction, $5^{\text {th }}$ ed., Addison-Wesley Publishing Company, USA, 2009.

[33] Shefelbine, S., Clarkson, J., Farmer, R., and Eason, S., Good Design Practice for Medical Devices and Equipment - Requirements Capture, University of Cambridge Engineering Design Centre and University of Cambridge Institute for Manufacturing, 2002.

[34] Wegman, E. J., and Luo, Q., "High Dimensional Clustering Using Parallel Coordinates and the Grand Tour," Classification and Knowledge Organization, edited by R. Klar and O. Opitz, Springer Berlin Heidelberg, 1997, pp. 93-101. 
[35] Heinrich, J., and Weiskopf, D., "Parallel Coordinates for Multidimensional Data Visualization: Basic Concepts," Computing in Science Engineering, Vol. 17, No. 3, 2015, pp. 70-76. https://doi.org/10.1109/MCSE.2015.55

[36] Artero, A. O., Oliveira, M. C. F. d., and Levkowitz, H., "Uncovering Clusters in Crowded Parallel Coordinates Visualizations," IEEE Symposium on Information Visualization, 2004, pp. 81-88. https://doi.org/10.1109/INFVIS.2004.68

[37] Carroll, J. M., and Carrithers, C., "Training wheels in a user interface," Communications of the ACM, Vol. 27, No. 8, 1984, pp. 800-806.

[38] Nielsen, J., “Usability Inspection Methods," Conference Companion on Human Factors in Computing Systems, ACM, New York, NY, USA, 1994, pp. 413-414. https://doi.org/10.1145/259963.260531

[39] Nielsen, J., "Enhancing the Explanatory Power of Usability Heuristics," Proceedings of the SIGCHI Conference on Human Factors in Computing Systems, ACM, New York, NY, USA, 1994, pp. 152-158. https://doi.org/10.1145/191666.191729

[40] Grossman, T., and Balakrishnan, R., “The Bubble Cursor: Enhancing Target Acquisition by Dynamic Resizing of the Cursor's Activation Area," Proceedings of the SIGCHI Conference on Human Factors in Computing Systems, ACM, 2005, pp. 281-290. https://doi.org/10.1145/1054972.1055012 event-place: Portland, Oregon, USA.

[41] Unity Game Engine, “Unity,” https://unity.com/, Last accessed: July 2018.

[42] Ankerst, M., Breunig, M. M., Kriegel, H.-P., and Sander, J., “OPTICS: Ordering Points to Identify the Clustering Structure,” SIGMOD Rec., Vol. 28, No. 2, 1999, pp. 49-60. https://doi.org/10.1145/304181.304187

[43] Ester, M., Kriegel, H.-P., Sander, J., and Xu, X., “A Density-based Algorithm for Discovering Clusters a Density-based Algorithm for Discovering Clusters in Large Spatial Databases with Noise," Proceedings of the Second International Conference on Knowledge Discovery and Data Mining, AAAI Press, 1996, pp. 226-231.

[44] Tory, M., and Moller, T., "Human factors in visualization research," IEEE transactions on visualization and computer graphics, Vol. 10, No. 1, 2004, pp. 72-84.

[45] Van Wijk, J. J., "Views on visualization,” IEEE transactions on visualization and computer graphics, Vol. 12, No. 4, 2006, pp. $421-432$.

[46] Kristensson, P. O., Dahlback, N., Anundi, D., Bjornstad, M., Gillberg, H., Haraldsson, J., Martensson, I., Nordvall, M., and Stahl, J., "An evaluation of space time cube representation of spatiotemporal patterns," IEEE Transactions on visualization and computer graphics, Vol. 15, No. 4, 2009, pp. 696-702.

[47] Ribeiro, M. T., Singh, S., and Guestrin, C., “"Why Should I Trust You?": Explaining the Predictions of Any Classifier,” CoRR, Vol. abs/1602.04938, 2016. URL http://arxiv.org/abs/1602.04938.

[48] Lundberg, S. M., and Lee, S.-I., “A Unified Approach to Interpreting Model Predictions,” Advances in Neural Information Processing Systems 30, edited by I. Guyon, U. V. Luxburg, S. Bengio, H. Wallach, R. Fergus, S. Vishwanathan, and R. Garnett, Curran Associates, Inc., 2017, pp. 4765-4774. 
[49] Ribeiro, M. T., Singh, S., and Guestrin, C., "Anchors: High-Precision Model-Agnostic Explanations," AAAI Publications, Thirty-Second AAAI Conference on Artificial Intelligence, 2018.

[50] Hettenhausen, J., Lewis, A., Randall, M., and Kipouros, T., "Interactive multi-objective particle swarm optimisation using decision space interaction," 2013 IEEE Congress on Evolutionary Computation, 2013, pp. 3411-3418. https://doi.org/10.1109/ CEC.2013.6557988 